Advances in Dynamical Systems and Applications

ISSN 0973-5321, Volume 14, Number 2, pp. 149-178 (2019)

https://dx.doi.org/10.37622/ADSA/14.2.2019.149-178

\title{
Stability and Periodicity of Certain Homogeneous Second-Order Fractional Difference Equations with Quadratic Terms
}

\author{
Mirela Garić-Demirović, Sabina Hrustić and Mehmed Nurkanović \\ University of Tuzla \\ Department of Mathematics \\ Tuzla, 75000, Bosnia and Herzegovina
}

\begin{abstract}
We investigate the local and global character of the unique equilibrium point of certain homogeneous fractional difference equation with quadratic terms. The existence of the period-two solution in one special case is given. Also, in this case the local and global stability of the minimal period-two solution for some special values of the parameters are given.
\end{abstract}

AMS Subject Classifications: 39A10, 39A20, 39A23, 39A30.

Keywords: Difference equations, equilibrium, attractivity, period-two solution, local stability, global stability, basin, stable manifold, unstable manifold.

\section{Introduction}

The following general second-order fractional difference equation with quadratic terms of the form

$$
x_{n+1}=\frac{A x_{n}^{2}+B x_{n} x_{n-1}+C x_{n-1}^{2}+D x_{n}+E x_{n-1}+F}{a x_{n}^{2}+b x_{n} x_{n-1}+c x_{n-1}^{2}+d x_{n}+e x_{n-1}+f}, \quad n=0,1, \ldots
$$

with nonnegative parameters and initial conditions such that $A+B+C>0, a+b+c+d+$ $e+f>0$ and $a x_{n}^{2}+b x_{n} x_{n-1}+c x_{n-1}^{2}+d x_{n}+e x_{n-1}+f>0, n=0,1, \ldots$ is an area of interest of mathematical researchers over last ten years. Several global asymptotic results for some special cases of Equation (1.1) were obtained in [4,5,8,9,16,21-23,25].

Received January 22, 2019; Accepted April 10, 2019

Communicated by Mustafa R. S. Kulenović 
One interesting special case of (1.1) is the following homogeneous linear fractional difference equation studied in $[3,11,12]$ :

$$
x_{n+1}=\frac{D x_{n}+E x_{n-1}}{d x_{n}+e x_{n-1}}, \quad n=0,1, \ldots
$$

which represents discretization of the differential equation model in biochemical networks, see [12]. Notice that equation (1.2) is also the special case of the linear fractional difference equation

$$
x_{n+1}=\frac{D x_{n}+E x_{n-1}+F}{d x_{n}+e x_{n-1}+f}, \quad n=0,1, \ldots
$$

(which was investigated in great detail in [11]) with well known but very complicated dynamics, such as Lyness' equation (see [13]).

Stability, periodicity and Neimark-Sacker bifurcation of the special case of (1.1), when $D=E=F=d=e=f=0$, i.e.,

$$
x_{n+1}=\frac{A x_{n}^{2}+B x_{n} x_{n-1}+C x_{n-1}^{2}}{a x_{n}^{2}+b x_{n} x_{n-1}+c x_{n-1}^{2}}, \quad n=0,1, \ldots
$$

was investigated in [8]. The following special cases of (1.4):

$$
x_{n+1}=\frac{x_{n-1}^{2}}{a x_{n}^{2}+b x_{n} x_{n-1}+c x_{n-1}^{2}}, \quad n=0,1, \ldots,
$$

and

$$
x_{n+1}=\frac{A x_{n}^{2}+C x_{n-1}^{2}}{a x_{n}^{2}+b x_{n} x_{n-1}}, \quad n=0,1, \ldots,
$$

were investigated in [4] and [5].

The first systematic study of global dynamics of a special case of Equation (1.1) where $A=C=D=a=c=d=0$ was performed in [1,2].

An interesting special case of Equation (1.1) where $A=C=F=a=c=$ $d=f=0$ was studied in [20]. This equation is an example of a rational difference equation, such that associated map is always strictly decreasing with respect to the second variable, and changes its monotonicity with respect to the first variable, i.e., can be increasing or decreasing depending on corresponding parametric space.

In this paper, we investigate the local and global character of the equilibrium point and the existence of period-two solutions of the following difference equation (which is the special case of (1.4) when $B=b=0$ ).

$$
u_{n+1}=\frac{A u_{n}^{2}+C u_{n-1}^{2}}{a u_{n}^{2}+c u_{n-1}^{2}},
$$

where the parameters $A, C, a, c$ are positive numbers and where the initial conditions $u_{-1}$ and $u_{0}$ are arbitrary nonnegative real numbers such that $u_{-1}+u_{0}>0$. 
Notice that the linear version of Equation (1.7) is Equation (1.2) which was considered in [12]. The authors proved that the unique equilibrium point of Equation (1.2) is globally asymptotically stable and that in some cases Equation (1.2) has only one locally stable period-two solution. If we replace linear with quadratic terms in Equation (1.2) we obtain a qualitative different dynamics. In particular, quadratic terms imply the existence of the locally stable period-two solution or two period-two solutions, one of them is locally stable and the other one is a saddle point, (i.e., Equation (1.7) has two period doubling bifurcations) as well as the existence of the minimal period-six solution. In the special case $a=0$, Equation (1.7) has very complicated behavior including chaos since quadratic terms imply also the phenomena of Neimark-Sacker bifurcation (see [10]).

\section{Preliminary Results}

By simple transformation $u_{n}=\frac{C}{c} x_{n}$, Equation (1.7) reduces to the equation

$$
x_{n+1}=\frac{A x_{n}^{2}+x_{n-1}^{2}}{a x_{n}^{2}+x_{n-1}^{2}},
$$

where the parameters $A, a$ are positive numbers and where the initial conditions $x_{-1}$ and $x_{0}$ are arbitrary nonnegative real numbers such that $x_{-1}+x_{0}>0$.

Equation (2.1) has a unique equilibrium point $\bar{x}=\frac{A+1}{a+1}$ and if we denote

$$
f(u, v)=\frac{A u^{2}+v^{2}}{a u^{2}+v^{2}}
$$

then

$$
f_{u}^{\prime}=\frac{2(A-a) u v^{2}}{\left(a u^{2}+v^{2}\right)^{2}}, f_{v}^{\prime}=-\frac{2(A-a) u^{2} v}{\left(a u^{2}+v^{2}\right)^{2}} .
$$

This means that right-hand side of Equation (2.1) is decreasing in $x_{n}$ and increasing in $x_{n-1}$ when $A<a$, and it is increasing in $x_{n}$ and decreasing in $x_{n-1}$ when $a<A$. In the case when $a<A$ for studying global stability of (2.1) we will use the following well-known result [13, Theorem 2.22].

Theorem 2.1. Let $[a, b]$ be a compact interval of real numbers and assume that $f$ : $[a, b] \times[a, b] \rightarrow[a, b]$ is a continuous function satisfying the following properties:

(a) $f(x, y)$ is non-decreasing in $x \in[a, b]$ for each $y \in[a, b]$, and $f(x, y)$ is nonincreasing in $y \in[a, b]$ for each $x \in[a, b]$;

(b) If $(m, M) \in[a, b] \times[a, b]$ is a solution of the system

$$
f(m, M)=m \quad \text { and } \quad f(M, m)=M,
$$

then $m=M$. 
Then

$$
x_{n+1}=f\left(x_{n}, x_{n-1}\right), \quad n=0,1, \ldots
$$

has a unique equilibrium $\bar{x} \in[a, b]$ and every solution of Equation (2.4) converges to $\bar{x}$.

The similar results are used in [18-20].

It can be shown that the Neimark-Sacker bifurcation does not exist in Equation (2.1) for $A>a>0$, but it was shown that Neimark-Sacker bifurcation occurs only in the special case when $a=0$, see [10]. However, in the case when $A<a$, there exists period-doubling bifurcation and that is the reason why we use the monotone maps techniques and the geometric techniques for studying global behavior of solutions of (2.1).

Let $I$ be some interval of real numbers and let $f \in C^{1}[I \times I, I]$, such that $\bar{x} \in I$, be an equilibrium point of a difference equation

$$
x_{n+1}=f\left(x_{n}, x_{n-1}\right), \quad x_{-1}, x_{0} \in I, \quad n=0,1, \ldots
$$

where $f$ is a continuous and decreasing in the first and increasing in the second variable. There are several global attractivity results for Equation (2.5) which give the sufficient conditions for all solutions to approach a unique equilibrium. These results were used efficiently in monograph [11] to study the global behavior of solutions of the secondorder linear fractional difference equation. One such result is:

Theorem 2.2. Let $[a, b]$ be an interval of real numbers and assume that $f:[a, b] \times$ $[a, b] \rightarrow[a, b]$ is a continuous function satisfying the following properties:

(a) $f(x, y)$ is non-increasing in first and non-decreasing in second variable.

(b) Equation (2.5) has no minimal period-two solutions in $[a, b]$.

Then every solution of Equation (2.5) converges to $\bar{x}$.

Throughout this paper we shall use the North-East ordering for which the positive cone is the first quadrant, i.e., this partial ordering is defined by $\left(x_{1}, y_{1}\right) \preceq_{n e}\left(x_{2}, y_{2}\right)$ if $x_{1} \leq x_{2}$ and $y_{1} \leq y_{2}$ and the South-East ordering defined as $\left(x_{1}, y_{1}\right) \preceq_{s e}\left(x_{2}, y_{2}\right)$ if $x_{1} \leq x_{2}$ and $y_{1} \geq y_{2}$.

The following result gives conditions for the existence of a global invariant curve through a hyperbolic or nonhyperbolic fixed point of a competitive map that separates regions with different dynamics [14, Theorem 1].

Theorem 2.3. Let $\mathrm{p}, \mathrm{q} \in \overline{\mathbb{R}}^{2}$ be such that $\mathrm{p} \ll_{\text {se }} \mathrm{q}$, and $\mathcal{R} \subset \mathbb{R}^{2}$ such that int $\left([\mathrm{p}, \mathrm{q}]_{\text {se }}\right) \subset$ $\mathcal{R} \subset[\mathrm{p}, \mathrm{q}]_{\text {se }}$. Let $T$ be a competitive map defined on $\mathcal{R}$ that is strongly competitive on int $(\mathcal{R})$. If there exist $\mathrm{r} \in\{\mathrm{p}, \mathrm{q}\}$, and $\mathrm{x}, \mathrm{y} \in \operatorname{int}(\mathcal{R})$ such that $T^{n}(\mathrm{x}) \rightarrow \mathrm{r}$ and $T^{n}(\mathrm{y}) \nrightarrow \mathrm{r}$, then there exists a curve $\mathcal{C}$ in $\mathcal{R}$ which is strongly north-east linearly ordered and whose endpoints are in $\partial \mathcal{R}$, such that the connected components $\mathcal{A}$ and $\mathcal{B}$ of int $(\mathcal{R}) \backslash \mathcal{C}$ chosen so that $\mathrm{x} \in \mathcal{A}$, satisfy $T^{n}(\mathrm{z}) \rightarrow \mathrm{r}$ for $\mathrm{z} \in \mathcal{A}$, and $T^{n}(\mathrm{w}) \nrightarrow \mathrm{r}$ for $\mathrm{w} \in \mathcal{B} \cup \mathcal{C}$. If the point $\mathrm{r}$ is in $\mathcal{R}$, then $\mathrm{r}$ is a fixed point of $T$. 
The next result gives sufficient conditions for the existence of at least one separatrix curve through a fixed point of a competitive map. For $x \in \mathbb{R}^{2}$, let $\mathcal{Q}_{l}(x), l=1, \ldots 4$ be the standard (closed) quadrants in $\mathbb{R}^{2}$ with respect to $x$ [14, Theorem 2].

Theorem 2.4. Let $\mathcal{R}=\left(a_{1}, a_{2}\right) \times\left(b_{1}, b_{2}\right)$ and let $T: \mathcal{R} \rightarrow \mathcal{R}$ be a strongly competitive map with a unique fixed point $\bar{x} \in \mathcal{R}$, and such that $T$ is twice continuously differentiable in a neighbourhood of $\bar{x}$. Assume further that at the point $\overline{\mathrm{x}}$ the map $T$ has associated characteristic values $\mu$ and $\nu$ satisfying $1<\mu$ and $-\mu<\nu<\mu$, with $\nu \neq 0$, and that no standard basis vector is an eigenvector associated to one of the characteristic values.

Then there exist curves $\mathcal{C}_{1}, \mathcal{C}_{2}$ in $\mathcal{R}$ and there exist $\mathrm{p}_{1}$, $\mathrm{p}_{2} \in \partial \mathcal{R}$ with $\mathrm{p}_{1} \ll_{\text {se }} \bar{x} \ll_{\text {se }}$ $\mathrm{p}_{2}$ such that:

(i) For $l=1,2, \mathcal{C}_{l}$ is invariant, north-east strongly linearly ordered, such that $\bar{x} \in \mathcal{C}_{l}$ and $\mathcal{C}_{l} \subset \mathcal{Q}_{3}(\bar{x}) \cup \mathcal{Q}_{1}(\bar{x}) ;$ the endpoints $\mathrm{q}_{l}, \mathrm{r}_{l}$ of $\mathcal{C}_{l}$, where $\mathrm{q}_{l} \ll_{n e} \mathrm{r}_{l}$, belong to the boundary of $\mathcal{R}$. For $l, j \in\{1,2\}$ with $l \neq j, \mathcal{C}_{l}$ is a subset of the closure of one of the components of $\mathcal{R} \backslash \mathcal{C}_{j}$. Both $\mathcal{C}_{1}$ and $\mathcal{C}_{2}$ are tangential at $\bar{x}$ to the eigenspace associated with $\nu$.

(ii) For $l=1,2$ let $\mathcal{B}_{l}$ be the component of $\mathcal{R} \backslash \mathcal{C}_{l}$ whose closure contains $\mathrm{p}_{l}$. Then $\mathcal{B}_{l}$ is invariant. Also, for $\mathrm{x} \in \mathcal{B}_{1}, T^{n}(x)$ accumulates on $\mathcal{Q}_{2}\left(\mathrm{p}_{1}\right) \cap \partial \mathcal{R}$, and for $\mathrm{x} \in \mathcal{B}_{2}, T^{n}(x)$ accumulates on $\mathcal{Q}_{4}\left(\mathrm{p}_{2}\right) \cap \partial \mathcal{R}$.

(iii) Let $\mathcal{D}_{1}:=\mathcal{Q}_{1}(\bar{x}) \cap \mathcal{R} \backslash\left(\mathcal{B}_{1} \cup \mathcal{B}_{2}\right)$ and $\mathcal{D}_{2}:=\mathcal{Q}_{3}(\bar{x}) \cap \mathcal{R} \backslash\left(\mathcal{B}_{1} \cup \mathcal{B}_{2}\right)$. Then $\mathcal{D}_{1} \cup \mathcal{D}_{2}$ is invariant.

In [4] and [5] the authors gave more precisely the dynamics in two special cases of (1.4) where the right-hand side of (1.4) is decreasing in $x_{n}$ and increasing in $x_{n-1}$ and where they could have applied the theory of monotone maps to give global dynamics. Also, see $[6,7,15-18,24]$ for an application of the monotone maps techniques to some competitive systems of fractional difference equations. In [15] very interesting geometric method for linearized stability analysis of some competitive maps was given. One such result is [15, Lemma 5]:

Lemma 2.5. Let $\mathcal{U}$ be a nonempty subset of $\mathbb{R}^{2}$, and $\left(F_{1}, F_{2}\right): \mathcal{U} \rightarrow \mathbb{R}^{2}$ be a continuously differentiable strongly competitive map. Let $(\bar{x}, \bar{y}) \in \mathcal{U}^{\circ}$ be an isolated fixed point of $\left(F_{1}, F_{2}\right)$. Set $\alpha:=\left(\partial F_{1} / \partial x\right)(\bar{x}, \bar{y})$ and $\delta:=\left(\partial F_{2} / \partial y\right)(\bar{x}, \bar{y})$, and let $\mathcal{C}_{1}=\left\{(x, y): F_{1}(x, y)=x\right\}$ and $\mathcal{C}_{2}=\left\{(x, y): F_{2}(x, y)=y\right\}$. Then,

(i) There exists an open interval $\mathcal{I} \subset \mathbb{R}$ containing $\bar{x}$ such that $\mathcal{C}_{1}$ and $\mathcal{C}_{2}$ are the graphs of differentiable and strictly decreasing functions $y_{1}(x)$ and $y_{2}(x)$ of $x \in$ $\mathcal{I}$ if and only if $\alpha<1$ and $\delta<1$.

(ii) Suppose that $\alpha<1$ and $\delta<1$. If the Jacobian matrix of $F=\left(F_{1}, F_{2}\right)$ at $(\bar{x}, \bar{y})$ does not have a negative unstable eigenvalue, then the following statements are true: 
(a) $(\bar{x}, \bar{y})$ is nonhyperbolic if and only if $y_{1}^{\prime}(\bar{x})=y_{2}^{\prime}(\bar{x})$;

(b) $(\bar{x}, \bar{y})$ is a saddle if and only if $y_{1}^{\prime}(\bar{x})>y_{2}^{\prime}(\bar{x})$;

(c) $(\bar{x}, \bar{y})$ is hyperbolic attractor if and only if $y_{1}^{\prime}(\bar{x})<y_{2}^{\prime}(\bar{x})$.

Now, we give the following result about local dynamics of Equation (2.1) (see Figure 2.1).

Theorem 2.6. Equation (2.1) has the unique positive equilibrium point $\bar{x}=\frac{A+1}{a+1}$.

1) If $(A, a) \in \Lambda_{1} \cup \Lambda_{2} \cup \Lambda_{3}$, where

$$
\begin{aligned}
& \Lambda_{1}=\left\{(A, a) \mid A \leq 1 \wedge a<\frac{5 A+1}{3-A}\right\} \\
& \Lambda_{2}=\left\{(A, a) \mid 1<A<3 \wedge \frac{A-1}{A+3}<a<\frac{5 A+1}{3-A}\right\}, \\
& \Lambda_{3}=\left\{(A, a) \mid A \geq 3 \wedge a>\frac{A-1}{A+3}\right\}
\end{aligned}
$$

then the equilibrium point $\bar{x}$ is locally asymptotically stable.

2) If $a<\frac{A-1}{A+3}$, then the equilibrium point $\bar{x}$ is a repeller.

3) If $A<3$ and $a>\frac{1+5 A}{3-A}$, then the equilibrium point $\bar{x}$ is a saddle point.

4) If $a=\frac{A-1}{A+3}$, then the equilibrium point $\bar{x}$ is nonhyperbolic of elliptic type with eigenvalues $\lambda_{1,2}=\frac{1 \pm i \sqrt{3}}{2}$.

5) If $a=\frac{1+5 A}{3-A}$ and $A \neq 3$ then the equilibrium point $\bar{x}$ is nonhyperbolic of stable type with eigenvalues $\lambda_{1,2} \in\left\{-1, \frac{1}{2}\right\}$.

Proof. Since the linearized equation associated with (2.1) about the equilibrium point $\bar{x}$ is of the form

$$
y_{n+1}=s y_{n}+t y_{n-1}
$$

where $s=\frac{\partial f}{\partial u}(\bar{x}, \bar{x}), t=\frac{\partial f}{\partial v}(\bar{x}, \bar{x})$ and

$$
s=-t=\frac{\partial f}{\partial u}(\bar{x}, \bar{x})=\left(\frac{2(A-a) u v^{2}}{\left(a u^{2}+v^{2}\right)^{2}}\right)(\bar{x}, \bar{x})=\frac{2(A-a)}{(A+1)(a+1)},
$$

then the corresponding characteristic equation at the equilibrium point is

$$
\lambda^{2}-s \lambda-t=0
$$

i) Equilibrium point $\bar{x}$ is locally asymptotically stable if

$$
\begin{aligned}
|s| & <1-t<2 \Longleftrightarrow|s|<1+s<2 \Longleftrightarrow-\frac{1}{2}<s<1 \\
& \Longleftrightarrow\{a(3-A)<5 A+1 \wedge a(A+3)>A-1\} \\
& \Longleftrightarrow\left\{\begin{array}{c}
\left(A \leq 1 \wedge a<\frac{5 A+1}{3-A}\right) \vee\left(1<A<3 \wedge \frac{A-1}{A+3}<a<\frac{5 A+1}{3-A}\right) \\
\vee\left(A \geq 3 \wedge a>\frac{A-1}{A+3}\right)
\end{array}\right\} .
\end{aligned}
$$


ii) Equilibrium point $\bar{x}$ is a saddle point if

$$
\begin{aligned}
\left\{|s|>|1-t| \wedge s^{2}+4 t>0\right\} & \Longleftrightarrow\left\{s^{2}>(1+s)^{2} \wedge s(s-4)>0\right\} \\
& \Longleftrightarrow s<-\frac{1}{2} \Longleftrightarrow\left\{A<3 \wedge a>\frac{1+5 A}{3-A}\right\}
\end{aligned}
$$

iii) Equilibrium point $\bar{x}$ is a repeller if

$$
\begin{gathered}
\{|s|<|1-t| \wedge|t|>1\} \Longleftrightarrow\left\{s^{2}<(1+s)^{2} \wedge|s|>1\right\} \Longleftrightarrow\left\{s>-\frac{1}{2} \wedge|s|>1\right\} \\
\Longleftrightarrow s>1 \Longleftrightarrow a(A+3)<A-1 \Longleftrightarrow a<\frac{A-1}{A+3} .
\end{gathered}
$$

iv) Equilibrium point $\bar{x}$ is nonhyperbolic if

$$
\begin{aligned}
\{|s|=|1-t| \vee(t=-1 \wedge|s| \leq 2)\} & \Longleftrightarrow\left\{s=-\frac{1}{2} \vee s=1\right\} \\
& \Longleftrightarrow\left\{\left(a=\frac{1+5 A}{3-A} \wedge A \neq 3\right) \vee a=\frac{A-1}{A+3}\right\} .
\end{aligned}
$$

If $s=1$, i.e., $a(A+3)=A-1$, then the characteristic equation (2.6) becomes

$$
\lambda^{2}-\lambda+1=0
$$

with eigenvalues $\lambda_{1,2}=\frac{1 \pm i \sqrt{3}}{2}$.

If $s=-\frac{1}{2}$, i.e., $a(3-A)=5 A+1$, then the characteristic equation (2.6) is of the form

$$
2 \lambda^{2}+\lambda-1=0
$$

with eigenvalues $\lambda_{1}=-1$ and $\lambda_{2}=\frac{1}{2}$.

Notice that

$$
\begin{aligned}
& x_{n+1}=\frac{A x_{n}^{2}+x_{n-1}^{2}}{a x_{n}^{2}+x_{n-1}^{2}} \leq \frac{\max \{A, 1\}\left(x_{n}^{2}+x_{n-1}^{2}\right)}{\min \{a, 1\}\left(x_{n}^{2}+x_{n-1}^{2}\right)}=\frac{\max \{A, 1\}}{\min \{a, 1\}}, \\
& x_{n+1}=\frac{A x_{n}^{2}+x_{n-1}^{2}}{a x_{n}^{2}+x_{n-1}^{2}} \geq \frac{\min \{A, 1\}\left(x_{n}^{2}+x_{n-1}^{2}\right)}{\max \{a, 1\}\left(x_{n}^{2}+x_{n-1}^{2}\right)}=\frac{\min \{A, 1\}}{\max \{a, 1\}}
\end{aligned}
$$

for $n=1,2,3, \ldots$. It means that $I=\left[\frac{\min \{A, 1\}}{\max \{a, 1\}}, \frac{\max \{A, 1\}}{\min \{a, 1\}}\right]$ is an invariant interval for the function $f(u, v)=\frac{A u^{2}+v^{2}}{a u^{2}+v^{2}}$ and attracting interval for solutions of Equation (2.1). 


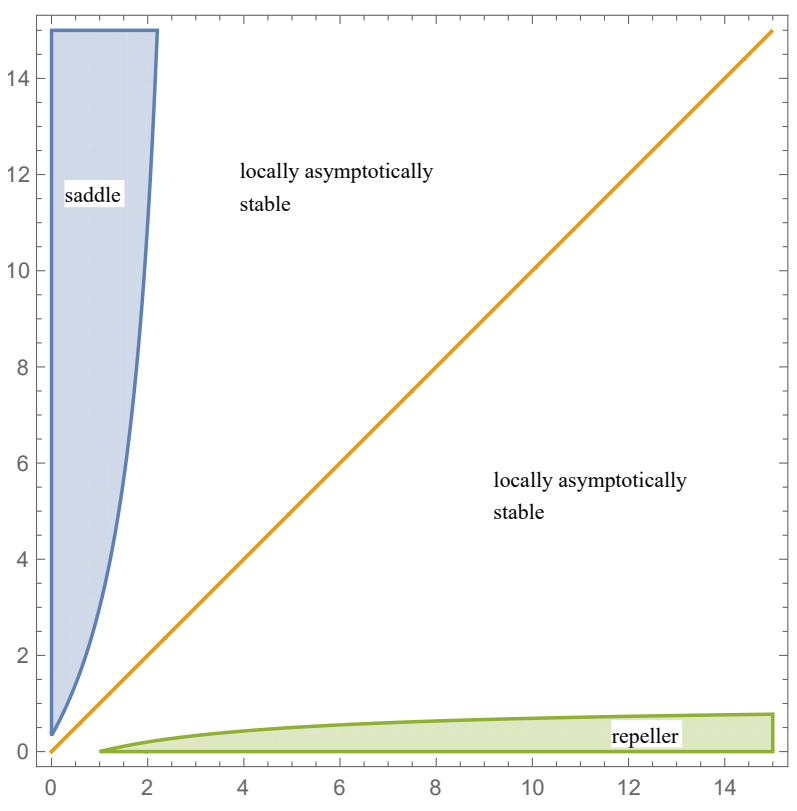

Figure 2.1: The areas of the parameters for each type of local stability

\section{Case $a<A$}

In this case, the function $f(u, v)=\frac{A u^{2}+v^{2}}{a u^{2}+v^{2}}$ is increasing in the first and decreasing in the second variable, i.e.,

$$
f_{u}^{\prime}>0 \text { and } f_{v}^{\prime}<0
$$

Since $I=\left[\frac{\min \{A, 1\}}{\max \{a, 1\}}, \frac{\max \{A, 1\}}{\min \{a, 1\}}\right]$ is an invariant interval for the function $f$ and attracting interval for solutions of Equation (2.1), we can apply Theorem 2.1. It means that we need to find conditions under which the system (2.3), i.e.,

$$
m=\frac{A m^{2}+M^{2}}{a m^{2}+M^{2}}, \quad M=\frac{A M^{2}+m^{2}}{a M^{2}+m^{2}}
$$

has the unique solution $(m, M)=(\bar{x}, \bar{x})$.

By subtracting the equations of System (3.1) we get the following equation

$$
(M-m)[a(m+M)-(a+1) m M-(A-1)(m+M)]=0,
$$

from which one solution is $(m, M)=(\bar{x}, \bar{x})$.

If we put

$$
m M=x, \quad m+M=y,
$$

we obtain that $M$ and $m$ are solutions of the quadratic equation

$$
t^{2}-y t+x=0
$$


If $\Delta=y^{2}-4 x<0$, then System (3.1) has only one solution, $(m, M)=(\bar{x}, \bar{x})$.

From (3.2) we obtain

$$
a y^{2}-(a+1) x-(A-1) y=0,
$$

or

$$
x=\frac{1}{a+1}\left(a y^{2}-(A-1) y\right) .
$$

Also, by adding the equations of System (3.1) we get the following equation

$$
a y^{3}+(1-3 a) x y-(1+A) y^{2}+2(A+1) x=0 .
$$

By substituting (3.5) into (3.6), we obtain the quadratic equation

$$
a(a-1) y^{2}+(A-2 A a+a) y+A^{2}-1=0,
$$

whose solutions are given as $y_{ \pm}=\frac{A(a-1)+a(A-1) \pm \sqrt{D(A, a)}}{2 a(a-1)}$, where $D(A, a)=(5-$ $4 A) a^{2}+2(A-2) a+A^{2}$.

We see that $D(A, a)=0$ for $a_{ \pm}=\frac{2-A \pm 2(1-A) \sqrt{1+A}}{5-4 A}$.

Remark 3.1. If $D(A, a)<0$, then System (3.1) has the unique solution $(m, M)=$ $(\bar{x}, \bar{x})$. But, if $D(A, a) \geq 0$, then System (3.1) has the unique solution $(m, M)=(\bar{x}, \bar{x})$ if $y_{ \pm}<0$ or $x_{ \pm}<0$ or $\Delta=y^{2}-4 x=\frac{y}{a+1}[(1-3 a) y+4(A-1)]<0$.

Also, notice that

$$
x>0 \Longleftrightarrow\left\{(A<1 \wedge y>0) \vee\left(A>1 \wedge y>\frac{A-1}{a}\right)\right\} .
$$

First, consider the situation when $a=1<A$. Then $D(A, 1)=(A-1)^{2} \geq 0$ for all $A>0$ and $x=y=A+1>0$ for $A \neq 1$. The condition $y^{2}-4 x=(A+1)(A-3)<0$ is satisfied for $1 \neq A<3$ and then $m, M \notin \mathbb{R}$, so that System (3.1) has the unique solution $(m, M)=(\bar{x}, \bar{x})$ if $(A, a) \in S_{1}=\{(A, a) \mid a=1<A<3\}$.

On the other hand, if $A=1$, then $y_{-}=0$ and $y_{+}=\frac{1}{a}, x_{+}=\frac{1}{a(a+1)}>0$, such that $y_{+}^{2}-4 x_{+}<0 \Longleftrightarrow \frac{1}{3}<a<A=1$. It means that System (3.1) has the unique solution $(m, M)=(\bar{x}, \bar{x})$ if $(A, a) \in S_{2}=\left\{(A, a) \mid A=1>a>\frac{1}{3}\right\}$.

Now, we investigate the existence of a unique solution of System (3.1) depending on the sign of term $D(A, a)$ when $a \neq 1$.

A) $D(A, a)<0$.

In this case, $y_{ \pm} \notin \mathbb{R}$ and System (3.1) has the unique solution $(m, M)=(\bar{x}, \bar{x})$. That is why we analyze for which values of the parameters $A$ and $a$ is $D(A, a)<0$.

i) If $A=\frac{5}{4}$, then

$$
D\left(\frac{5}{4}, a\right)=-\frac{3}{2} a+\frac{25}{16}<0 \Longleftrightarrow \frac{5}{4}=A>a>\frac{25}{24} .
$$


ii) If $A>\frac{5}{4}$, then $a_{-}<0, a_{+}>0$ and $D(A, a)<0$ which means that $(A, a) \in S_{3}=$ $\left\{(A, a) \mid A>\frac{5}{4} \wedge a_{+}<a<A\right\}$.

iii) If $A<\frac{5}{4}$, then $a_{-}>0, a_{+}>0$. Also, $D(A, a)<0$ if $\min \left\{a_{-}, a_{+}\right\}<a<$ $\max \left\{a_{-}, a_{+}\right\}$. Since $a_{-}<A<a_{+}$for $A<1$ and $a_{+}<A<a_{-}$for $1<A<\frac{5}{4}$, we see that $D(A, a)<0 \Longleftrightarrow(A, a) \in S_{4} \cup S_{5}$, where

$$
S_{4}=\left\{(A, a) \mid A<1 \wedge a_{-}<a<A\right\}, S_{5}=\left\{(A, a) \mid 1<A<\frac{5}{4} \wedge a_{+}<a<A\right\} .
$$

B) $D(A, a)=0$.

a) If $A=\frac{5}{4}$, then $a=\frac{25}{24}$. This and (3.9) mean that System (3.1) has the unique solution $(m, M)=(\bar{x}, \bar{x})$ when $(A, a) \in S_{6}=\left\{(A, a) \mid \frac{25}{24} \leq a<A=\frac{5}{4}\right\}$.

b) If $A>\frac{5}{4}$, then $a_{-}<0, a_{+}>1$ and $D(A, a)=0$ for $a=a_{+}$, so Equation (3.7) has one positive solution $y_{+}$. Since $y_{+}>\frac{A-1}{a}$, then $x_{+}>0$. Now,

$$
\begin{aligned}
y^{2}-4 x<0 & \Longleftrightarrow(1-3 a) y_{-}<4(1-A) \Longleftrightarrow(2 A-5) a^{2}-(3 A-7) a-A<0 \\
& \Longleftrightarrow\left(\frac{5}{2}<A \wedge a_{+}<a^{+}\right) \vee\left(\frac{5}{4}<A \leq \frac{5}{2} \wedge a_{+}>a^{+}\right)
\end{aligned}
$$

where $a^{+}=\frac{3 A-7+\sqrt{17 A^{2}-62 A+49}}{2(2 A-5)}$, and which means that $y^{2}-4 x<0 \Longleftrightarrow \frac{31+8 \sqrt{2}}{17}<$ $A<5+2 \sqrt{5}$. This implies that System (3.1) has a unique solution $(m, M)=(\bar{x}, \bar{x})$ when $(A, a) \in S_{7}=\left\{(A, a) \mid \frac{31+8 \sqrt{2}}{17}<A<5+2 \sqrt{5} \wedge a=a_{+}\right\}$.

c) If $A<\frac{5}{4}$, then $a_{-}>0, a_{+}>0$ and $D(A, a)=0$ for $a=a_{+}$. It is easy to see that $a_{+}<A<a_{-}$for $A \in\left(1, \frac{5}{4}\right)$ and that $y=y_{+}<0$. This means that System (3.1) has the unique solution $(m, M)=(\bar{x}, \bar{x})$ when $(A, a) \in S_{8}=\left\{(A, a) \mid 1<A<\frac{5}{4} \wedge a=a_{+}\right\}$. If $A<1$, then $a_{-}<A<a_{+}$and $y_{-}>0, x_{-}>0$. The condition (3.10) is satisfied for $1>A \geq \frac{7}{9}$, but for $A<\frac{7}{9}$ we have that

$$
\begin{aligned}
& \Longleftrightarrow y_{-}<\frac{4(1-A)}{1-3 a} \Longleftrightarrow(2 A-5) a^{2}-(3 A-7) a-A<0 \\
& \Longleftrightarrow(A, a) \in S_{9}=\left\{(A, a) \mid A<1 \wedge a=a_{-}\right\}
\end{aligned}
$$

which means that System (3.1) has the unique solution $(m, M)=(\bar{x}, \bar{x})$.

C) $D(A, a)>0$.

I) $A=\frac{5}{4}$

In this case, $D(A, a)>0$ if $a<\frac{25}{24}$.

If $a<1$, then Equation (3.7) has one positive solution $y_{-}$and since $y_{-}>\frac{A-1}{a}$ it implies that $x_{-}>0$. The condition $y^{2}-4 x<0 \Longleftrightarrow(1-3 a) y_{-}<-1$ is satisfied for $a \in\left(\frac{11}{25}, 1\right)$. On the other hand, if $a>1$, then Equation (3.7) has one positive solution $y_{+}$, and since $y_{+}>\frac{A-1}{a}$ it implies $x_{+}>0$. The condition $(1-3 a) y_{+}<-1$ is satisfied for $a \in\left(1, \frac{25}{24}\right)$. This implies that System (3.1) has the unique solution $(m, M)=(\bar{x}, \bar{x})$ when $(A, a) \in S_{10}=\left\{(A, a) \mid A=\frac{5}{4} \wedge a \in\left(\frac{11}{25}, 1\right) \cup\left(1, \frac{25}{24}\right)\right\}$. 
II) $A>\frac{5}{4}$

In this case, $a_{-}<0, a_{+}>1$ and $D(A, a)>0$ if $a \in\left(0, a_{+}\right)$.

Suppose that $a<1$. For these conditions, Equation (3.7) has one positive solution $y_{-}$, and since

$$
y_{-}>\frac{A-1}{a} \Longleftrightarrow a+A-2<D(A, a) \Longleftrightarrow(A-1)\left(a^{2}-1\right)<0,
$$

we conclude that $x_{-}>0$. Now, we check the following condition

$$
y^{2}-4 x<0 \Longleftrightarrow(1-3 a) y_{-}<4(1-A) .
$$

If $a<\frac{1}{3}$, then (3.10) is not satisfied and System (3.1) has three positive solutions. If $a>\frac{1}{3}$, then (3.10) is satisfied for

$$
(A, a) \in S_{11}=\left\{(A, a) \mid \frac{5}{4}<A<3 \wedge \frac{3 A-1}{A+5}<a<1\right\}
$$

and System (3.1) has the unique solution $(m, M)=(\bar{x}, \bar{x})$.

If $a>1$, then Equation (3.7) has two positive solutions $y_{-}$and $y_{+}, y_{-}<y_{+}$, and since $y_{ \pm}>\frac{A-1}{a}$, we conclude that $x_{ \pm}>0$. The condition (3.10) is of the form

$$
y_{-}>\frac{4(1-A)}{1-3 y} \Longleftrightarrow(A, a) \in S_{12} \cup S_{13},
$$

where

$$
\begin{gathered}
S_{12}=\left\{(A, a) \mid \frac{5}{4}<A \leq 3 \wedge 1<a<a_{+}\right\}, \\
S_{13}=\left\{(A, a) \mid 3<A<5+2 \sqrt{5} \wedge \frac{3 A-1}{A+5}<a<a_{+}\right\} .
\end{gathered}
$$

III) $A<\frac{5}{4}$

In this case, $a_{-}>0, a_{+}>0$ and $D(A, a)>0$ for $a \in\left(0, a_{-}\right) \cup\left(a_{-}, a_{+}\right)$.

Assume that $a<1$ and $A \in\left(1, \frac{5}{4}\right)$. Under these conditions Equation (3.7) has one positive solution $y_{-}$, and since $y_{-}>\frac{A-1}{a}$ we obtain that $x_{-}>0$. This implies that System (3.1) has the unique solution $(m, M)=(\bar{x}, \bar{x})$ when

$$
y_{-}>\frac{4(1-A)}{1-3 a} \Longleftrightarrow(A, a) \in S_{14}=\left\{(A, a) \mid 1<A<\frac{5}{4} \wedge \frac{3 A-1}{A+5}<a<1\right\} .
$$

If $a>1$ and $A \in\left(1, \frac{5}{4}\right)$, then Equation (3.7) has two positive solutions $y_{-}$and $y_{+}, y_{-}<$ $y_{+}$, and since $y_{ \pm}>\frac{A-1}{a}$, it implies that $x_{ \pm}>0$. System (3.1) has the unique solution $(m, M)=(\bar{x}, \bar{x})$ if

$$
y_{-}>\frac{4(1-A)}{1-3 a} \Longleftrightarrow(A, a) \in S_{15}=\left\{(A, a) \mid 1<A<\frac{5}{4} \wedge 1<a<a_{+}\right\} .
$$


If $a<1$ and $A<1$, then Equation (3.7) has two positive solutions $y_{-}$and $y_{+}, y_{+}<y_{-}$, and since $y_{ \pm}>0$, it implies that $x_{ \pm}>0$. For $a \leq \frac{1}{3}$ condition (3.10) is satisfied if

$$
y_{-}<\frac{4(1-A)}{1-3 a} \Longleftrightarrow(A, a) \in S_{16} \cup S_{17},
$$

where

$$
\begin{aligned}
& S_{16}=\left\{(A, a) \mid 5-2 \sqrt{5}<A \leq \frac{7}{9} \wedge \frac{3 A-1}{A+5}<a<a_{-}\right\} \\
& S_{17}=\left\{(A, a) \mid \frac{7}{9}<A<1 \wedge \frac{3 A-1}{A+5}<a \leq \frac{1}{3}\right\} .
\end{aligned}
$$

For $a \in\left(\frac{1}{3}, 1\right)$ condition (3.10) is satisfied if

$$
y_{ \pm}>\frac{4(1-A)}{1-3 a} \Longleftrightarrow(A, a) \in S_{18}=\left\{(A, a) \mid \frac{7}{9}<A<1 \wedge \frac{1}{3}<a<a_{-}\right\} .
$$

Remark 3.2. Notice that $\bigcup_{i=1}^{18} S_{i}=\bigcup_{i=1}^{5} \Omega_{i}$, where

$$
\begin{aligned}
& \Omega_{1}=\left\{(A, a) \mid 0<A<5-2 \sqrt{5} \wedge a_{-} \leq a<A\right\}, \\
& \Omega_{2}=\left\{(A, a) \mid 5-2 \sqrt{5}<A \leq \frac{5}{4} \wedge \frac{3 A-1}{A+5}<a<A\right\}, \\
& \Omega_{3}=\left\{(A, a) \mid \frac{5}{4}<A \leq \frac{31+8 \sqrt{2}}{17} \wedge \frac{3 A-1}{A+5}<a<a_{+} \vee a_{+}<a<A\right\}, \\
& \Omega_{4}=\left\{(A, a) \mid \frac{31+8 \sqrt{2}}{17}<A \leq 5+2 \sqrt{5} \wedge \frac{3 A-1}{A+5}<a<A\right\}, \\
& \Omega_{5}=\left\{(A, a) \mid A>5+2 \sqrt{5} \wedge a_{+}<a<A\right\} .
\end{aligned}
$$

See Figure 3.1.

Theorem 3.3. If $(A, a) \in \bigcup_{i=1}^{5} \Omega_{i}$, where $\Omega_{i}(i=1, \ldots, 5)$ are given in Remark 3.2, then equilibrium point $\bar{x}$ is globally asymptotically stable.

Proof. We have already proved that the next claim is valid: If $(A, a) \in \bigcup_{i=1}^{5} \Omega_{i}$, where $\Omega_{i}$ $(i=1, \ldots, 5)$ are given in Remark 3.2, then the system of the algebraic equations (2.3) has the unique solution $(m, M)=(\bar{x}, \bar{x})$. Since $\frac{A-1}{A+3}<\min \left\{\frac{3 A-1}{A+5}, a_{+}\right\}$, then by using Theorem 2.1 we conclude that $\bar{x}$ is globally asymptotically stable. 


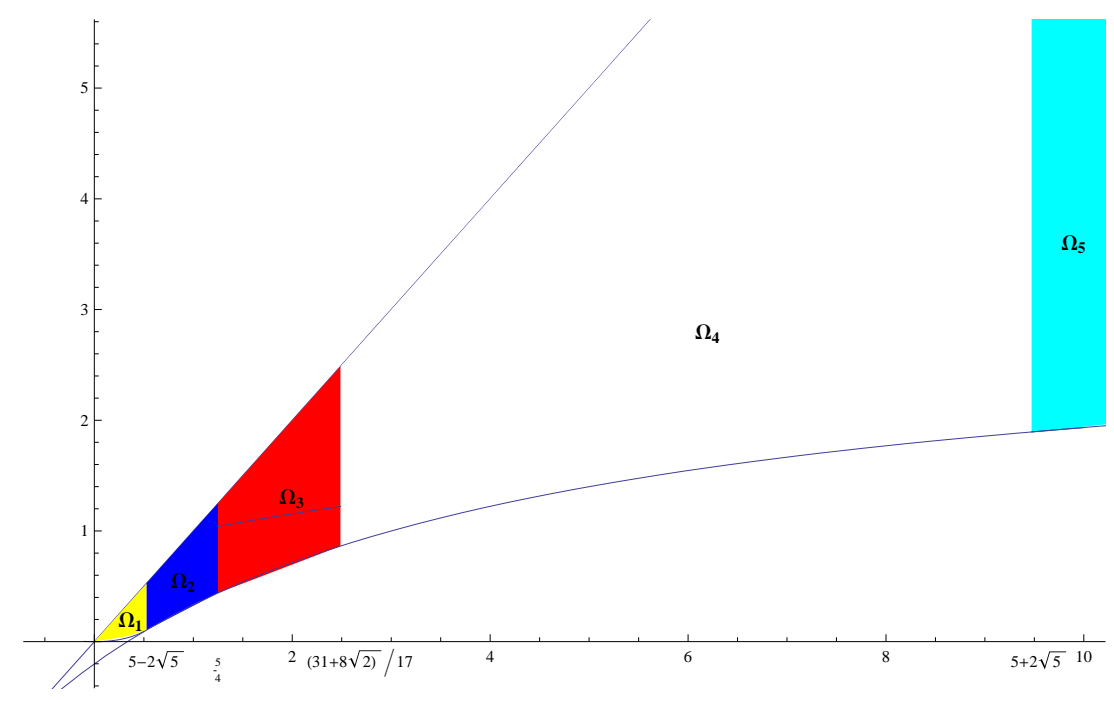

Figure 3.1: Visual representation of $\Omega_{i}-$ areas from Theorem 3.3

Based on many numerical simulations we made, we believe that the following conjecture is true.

Conjecture 3.4. If $(A, a) \in\left\{(A, a) \mid \frac{A-1}{A+3} \leq a<A\right\}$, then the equilibrium point $\bar{x}$ is globally asymptotically stable.

There is a particularly interesting situation in case $A>a$, when $\bar{x}$ is a repeller, and when each solution converges to a minimal period-six solution. Namely, many of our simulations clarify the following conjecture.

Conjecture 3.5. If $0<a<\frac{A-1}{A+3}$, then every nonequilibrium solution of Equation (2.1) converges to a minimal period-six solution.

The situation described in Conjecture 3.5 occurs for example when $A=3$ and $a=0.3$ in Equation (2.1). See Figure 3.2.

Remark 3.6. Straightforward calculation shows that there does not exist the NeimarkSacker bifurcation in the Equation (2.1) for $a>0$, but it was shown that there exist the Neimark-Sacker bifurcation only in the special case when $a=0$, see [10].

\section{Case $a>A$}

In this case, $f$ is decreasing in the first and increasing in the second variable. 


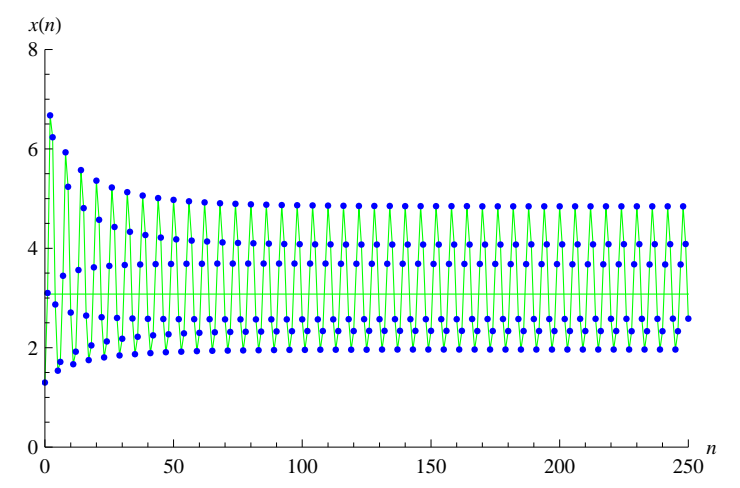

(a)

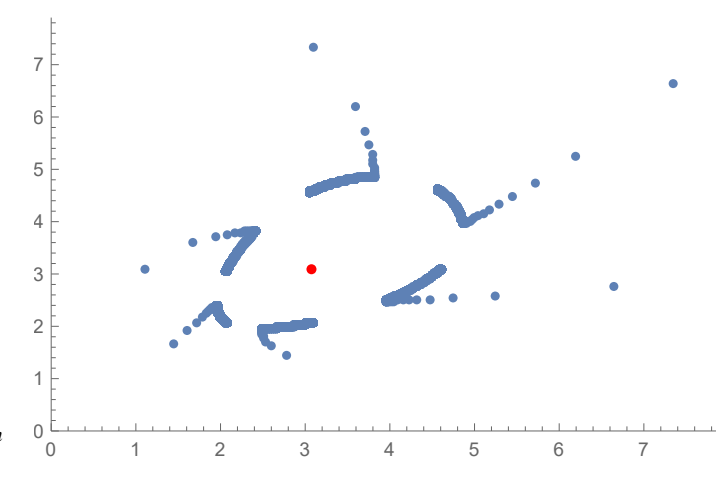

(b)

Figure 3.2: The phase portrait and the orbit for $A=3, a=0.3$ with initial values $\left(x_{-1}, x_{0}\right)=(1.1,3.1)$.

\subsection{Existence of Period-two Solutions}

Assume that $(\phi, \psi)$ is a minimal period-two solution of Equation (2.1) with $\phi, \psi \in$ $[0,+\infty)$ and $\phi \neq \psi$. Then

$$
\phi=\frac{A \psi^{2}+\phi^{2}}{a \psi^{2}+\phi^{2}}, \quad \psi=\frac{A \phi^{2}+\psi^{2}}{a \phi^{2}+\psi^{2}},
$$

i.e.,

$$
\phi\left(a \psi^{2}+\phi^{2}\right)=A \psi^{2}+\phi^{2}, \quad \psi\left(a \phi^{2}+\psi^{2}\right)=A \phi^{2}+\psi^{2}
$$

If we set

$$
\phi \psi=x \text { and } \phi+\psi=y,
$$

where $x>0$ and $y>0$, then $\phi$ and $\psi$ are positive and different solutions of the quadratic equation

$$
t^{2}-y t+x=0
$$

In addition to the conditions $x, y>0$, it is necessary that $y^{2}-4 x>0$. We obtain the following system

$$
\begin{aligned}
& -(a+1) x=y(1-A-y), \\
& x[(a-1) y+A+1]=A y^{2},
\end{aligned}
$$

from which we get

$$
(a-1) y^{2}+[-A(a+1)+(A+1)-(1-A)(a-1)] y-\left(1-A^{2}\right)=0 .
$$

I) If $a=1$, we get $x=A(A+1)>0, y=A+1>0$ and $y^{2}-4 x=(A+1)^{2}-$ $4 A(A+1)=(A+1)(1-3 A)>0$ for $A<\frac{1}{3}$. Therefore, if $a=1$ and $A<\frac{1}{3}$, then there exists a unique minimal period-two solution $(\phi, \psi)$, where

$$
\phi=\frac{1}{2}(A+1+\sqrt{(A+1)(1-3 A)}) \text { and } \psi=\frac{1}{2}(A+1-\sqrt{(A+1)(1-3 A)}) .
$$


II) Now suppose that $a \neq 1$. The roots of (4.3) are of the form $y_{ \pm}=\frac{-F \pm \sqrt{D}}{2(a-1)}$, where $F=2-a-A$ and $D=(2-a-A)^{2}+4(a-1)\left(1-A^{2}\right)$.

Notice that from (4.2) we have $t_{ \pm}=\frac{y}{2}\left(1 \pm \sqrt{\frac{(a-1) y-3 A+1}{(a-1) y+A+1}}\right)$, i.e., (2.1) has one or two minimal period-two solutions of the form

$$
\phi_{+}=\frac{y_{+}}{2}\left(1+\sqrt{\frac{(a-1) y_{+}-3 A+1}{(a-1) y_{+}+A+1}}\right), \quad \psi_{+}=\frac{y_{+}}{2}\left(1-\sqrt{\frac{(a-1) y_{+}-3 A+1}{(a-1) y_{+}+A+1}}\right),
$$

or

$$
\phi_{-}=\frac{y_{-}}{2}\left(1+\sqrt{\frac{(a-1) y_{-}-3 A+1}{(a-1) y_{-}+A+1}}\right), \quad \psi_{-}=\frac{y_{-}}{2}\left(1-\sqrt{\frac{(a-1) y_{-}-3 A+1}{(a-1) y_{-}+A+1}}\right) .
$$

The condition $y>0$ is satisfied in the following cases:

1. $\{A<1, a>1\} \Rightarrow y_{+}>0\left(y_{-}<0\right)$,

2. $\{A>1, a>1, D>0, F<0\} \Rightarrow y_{+}>y_{-}>0$,

3. $\{A<1, a<1, D>0, F>0\} \Rightarrow y_{-}>y_{+}>0$,

4. $\{A>1, a<1\} \Rightarrow y_{-}>0\left(y_{+}<0\right)$,

5. $\{D=0, \operatorname{sgn} F=-\operatorname{sgn}(a-1)\} \Rightarrow y_{+}=y_{-}=\frac{-F}{2(a-1)}>0$,

6. $\{A=1, \operatorname{sgn} F=-\operatorname{sgn}(a-1)\} \Rightarrow y_{-}=1\left(y_{+}<0\right)$.

Notice that the conditions $a<1$ and $A<1$ imply $F=2-a-A>2-1-A=1-A>0$ and the conditions $A>1$ and $a>1$ imply $F=2-a-A<2-1-A=1-A<0$. If $A=1$ than $F=-(a-1)$, so $\operatorname{sgn} F=-\operatorname{sgn}(a-1)$ is satisfied. Conditions $x>0$ and $y^{2}-4 x>0$ reduce to

$$
(a-1) y>3 A-1 .
$$

If $A>1$ and $a-1<0$, then the condition $(a-1) y_{-}>3 A-1=3(A-1)+2$ is not satisfied, so the case 4 . is impossible. In the next analysis we assume that $A \neq 1$. The case $A=1$ we will consider separately. After previous conclusions we have the following cases

1. $\{A<1, a>1\} \Rightarrow y_{+}>0\left(y_{-}<0\right)$,

2. $\{A>1, a>1, D>0\} \Rightarrow y_{+}>y_{-}>0$,

3. $\{A<1, a<1, D>0\} \Rightarrow y_{-}>y_{+}>0$,

4. $\{D=0, \operatorname{sgn} F=-\operatorname{sgn}(a-1)\} \Rightarrow y_{+}=y_{-}=\frac{-F}{2(a-1)}>0$.

Now, we will check when the inequality $(a-1) y_{+}>3 A-1$ is satisfied. Notice that

$$
\begin{aligned}
(a-1) y_{+}>3 A-1 & \Longleftrightarrow(a-1) \frac{-F+\sqrt{D}}{2(a-1)}>3 A-1 \Longleftrightarrow-F+\sqrt{D}>6 A-2 \\
& \Longleftrightarrow-2+a+A+\sqrt{D}>6 A-2 \Longleftrightarrow \sqrt{D}>5 A-a,
\end{aligned}
$$


which is true for $a \geq 5 A$ or $\left(a<5 A\right.$ and $\left.D>(5 A-a)^{2}\right)$ i.e.,

$$
(a \geq 5 A) \vee\left(\frac{5 A+1}{3-A}<a<5 A \wedge A<3\right) \text {. }
$$

Also,

$$
\begin{aligned}
(a-1) y_{-}>3 A-1 & \Longleftrightarrow(a-1) \frac{-F-\sqrt{D}}{2(a-1)}>3 A-1 \Longleftrightarrow-F-\sqrt{D}>6 A-2 \\
& \Longleftrightarrow-2+a+A-\sqrt{D}>6 A-2 \Longleftrightarrow \sqrt{D}<a-5 A,
\end{aligned}
$$

which is satisfied only for $a<5 A$ and $D<(5 A-a)^{2}$, i.e.,

$$
\begin{gathered}
a>5 A \wedge\left(A \geq 3 \vee\left(a<\frac{5 A+1}{3-A} \wedge A<3\right)\right) \\
\Longleftrightarrow(a>5 A \wedge A \geq 3) \vee\left(5 A<a<\frac{5 A+1}{3-A} \wedge A<3\right) .
\end{gathered}
$$

We can write: $D=a^{2}\left[\left(2 \frac{1}{a}-1-\frac{1}{a} A\right)^{2}+4 \frac{1}{a}\left(1-\frac{1}{a}\right)\left(1-A^{2}\right)\right]$. By substitution $\frac{1}{a}=$ $t$ we get $D=a^{2}\left[A(5 A-4) t^{2}+2 A(1-2 A) t+1\right]$. The solutions of the equation $D=0$ are $t=-\frac{1}{2 A(1-2 A)}$, for $A=\frac{4}{5}$, and $t_{ \pm}=\frac{A(2 A-1) \pm 2 \sqrt{A(A+1)(A-1)^{2}}}{A(5 A-4)}$, for $A \neq \frac{4}{5}$. Notice: if $A>\frac{4}{5}$, then $t_{-}<t_{+}$, and if $A<\frac{4}{5}$, then $t_{+}<t_{-}$, which implies

$$
\begin{aligned}
& \text { if } A>\frac{4}{5} \text {, then } D>0 \text { for } t<t_{-} \vee t>t_{+} \text {, } \\
& \text { if } A<\frac{4}{5} \text {, then } D>0 \text { for } t_{+}<t<t_{-} .
\end{aligned}
$$

Also, notice

$$
\begin{aligned}
\left(\frac{5 A+1}{3-A} \leq 5 A \wedge A<3\right) & \Longleftrightarrow A \in\left[1-\frac{2 \sqrt{5}}{5}, 1+\frac{2 \sqrt{5}}{5}\right] \\
A \geq 3 \vee\left(a<\frac{5 A+1}{3-A} \wedge A<3\right) & \Longleftrightarrow A \in\left(0,1-\frac{2 \sqrt{5}}{5}\right) \cup\left(1+\frac{2 \sqrt{5}}{5},+\infty\right)
\end{aligned}
$$

By straightforward calculation the previous cases reduce to the following two cases (I and II).

I) The equilibrium point $\bar{x}$ is a saddle and there exists one minimal period-two solution for $y_{+}$in the next cases:

1. $\left(1-\frac{2 \sqrt{5}}{5} \leq A \leq \frac{1}{3} \wedge 1<a\right) \vee\left(\frac{1}{3}<A<1 \wedge \frac{5 A+1}{3-A}<a\right)$

2. $1<A \leq 1+\frac{2 \sqrt{5}}{5} \wedge \frac{5 A+1}{3-A}<a$, 
3. $1-\frac{2 \sqrt{5}}{5} \leq A<\frac{1}{3} \wedge \frac{5 A+1}{3-A}<a<1$.

II) (a) Also, the equilibrium point $\bar{x}$ is saddle and there exists one minimal period-two solution for $y_{+}$in the next cases:

1. $A<1-\frac{2 \sqrt{5}}{5} \wedge 1<a$,

2. $1+\frac{2 \sqrt{5}}{5}<A<3 \wedge \frac{5 A+1}{3-A}<a$,

3. $A<1-\frac{2 \sqrt{5}}{5} \wedge \frac{5 A+1}{3-A}<a<1$.

(b) The equilibrium point $\bar{x}$ is locally asymptotically stable and then there exist two minimal period-two solutions in the following cases:

1. $\left(1+\frac{2 \sqrt{5}}{5}<A \leq 3 \wedge \frac{3-A}{5 A+1}<\frac{1}{a}<\frac{2 A-1}{5 A-4}-2 \sqrt{\frac{1-A-A^{2}+A^{3}}{A(5 A-4)^{2}}}\right)$ or $\left(A>3 \wedge \frac{1}{a}<\frac{2 A-1}{5 A-4}-2 \sqrt{\frac{1-A-A^{2}+A^{3}}{A(5 A-4)^{2}}}\right)$,

2. $A<1-\frac{2 \sqrt{5}}{5} \wedge \frac{3-A}{5 A+1}<\frac{1}{a}<\frac{2 A-1}{5 A-4}+2 \sqrt{\frac{1-A-A^{2}+A^{3}}{A(5 A-4)^{2}}}$,

3. $\left(A<1-\frac{2 \sqrt{5}}{5} \wedge\right.$ and $\left.\frac{1}{a}=\frac{2 A-1}{5 A-4}-2 \frac{1-A}{5 A-4} \sqrt{1+\frac{1}{A}}\right)$ or $\left(A>1+\frac{2 \sqrt{5}}{5}\right.$ and $\left.\frac{1}{a}=\frac{2 A-1}{5 A-4}+2 \frac{1-A}{5 A-4} \sqrt{1+\frac{1}{A}}\right) ; \quad y_{-}=y_{+}$.

Notice, if $a=\frac{5 A+1}{3-A}$, then

$$
a \geq 5 A \Leftrightarrow \frac{5 A+1}{3-A} \geq 5 a \Longleftrightarrow A \in\left(0,1-\frac{2 \sqrt{5}}{5}\right] \cup\left[1+\frac{2 \sqrt{5}}{5},+\infty\right)
$$

and by using (4.9) and (4.10), we conclude that Equation (2.1) has the unique minimal period-two solution $(\phi, \psi)=\left(\phi_{+}, \psi_{+}\right)$.

Merging previous cases we get the following Table 4.1. Also, see Figure 4.1. 


\begin{tabular}{|c|c|}
\hline Conditions & Minimal period-two solution(s) \\
\hline $\begin{array}{c}\left(A \leq \frac{1}{3} \text { and } 1<a\right) \\
\text { or } \\
\left(\frac{1}{3}<A<1 \text { and } \frac{5 A+1}{3-A}<a\right) \\
\left(1<A<3 \text { and } \frac{5 A+1}{3-A}<a\right) \\
\quad \text { or }\left(A<\frac{1}{3} \text { and } \frac{5 A+1}{3-A}<a<1\right) \\
\text { or } \\
\left(a=\frac{5 A+1}{3-A} \text { and } 0<A \leq \frac{5-2 \sqrt{5}}{5}\right) \\
\left(a=\frac{5 A+1}{3-A} \text { and } \frac{5+2 \sqrt{5}}{5} \leq A<3\right)\end{array}$ & $\begin{array}{l}\phi=\frac{y_{+}}{2}\left(1+\sqrt{\frac{(a-1) y_{+}-3 A+1}{(a-1) y_{+}+A+1}}\right) \\
\psi=\frac{y_{+}}{2}\left(1-\sqrt{\frac{(a-1) y_{+}-3 A+1}{(a-1) y_{+}+A+1}}\right)\end{array}$ \\
\hline$A=1$ and $a>3$ & $\begin{aligned} \phi & =\frac{1}{2}\left(1+\sqrt{\frac{a-3}{a+1}}\right) \\
\psi & =\frac{1}{2}\left(1-\sqrt{\frac{a-3}{a+1}}\right)\end{aligned}$ \\
\hline$A<\frac{1}{3}$ and $a=1$ & $\begin{array}{l}\phi=\frac{A+1+\sqrt{(A+1)(1-3 A)}}{2} \\
\psi=\frac{A+1+\sqrt{(A+1)(1-3 A)}}{2}\end{array}$ \\
\hline $\begin{array}{r}\left(A<\frac{5-2 \sqrt{5}}{5} \text { and } \frac{1}{a}=\eta_{-}(A)\right) \\
\text { or } \\
\left(A>\frac{5+2 \sqrt{5}}{5} \text { and } \frac{1}{a}=\eta_{+}(A)\right)\end{array}$ & $\begin{array}{l}\phi=\frac{a+A-2}{4(a-1)}\left(1+\sqrt{\frac{a-5 A}{a+3 A}}\right) \\
\psi=\frac{a+A-2}{4(a-1)}\left(1-\sqrt{\frac{a-5 A}{a+3 A}}\right)\end{array}$ \\
\hline $\begin{array}{c}\left(A<\frac{5-2 \sqrt{5}}{5} \text { and } \frac{3-A}{5 A+1}<\frac{1}{a}<\eta_{-}(A)\right) \\
\left.\text { or } \frac{5+2 \sqrt{5}}{5}<A \leq 3 \text { and } \frac{3-A}{5 A+1}<\frac{1}{a}<\eta_{+}(A)\right) \\
\text { or } \\
\left(A>3 \text { and } \frac{1}{a}<\eta_{+}(A)\right)\end{array}$ & $\begin{array}{l}\phi_{+}=\frac{y_{+}}{2}\left(1+\sqrt{\frac{(a-1) y_{+}-3 A+1}{(a-1) y_{+}+A+1}}\right) \\
\psi_{+}=\frac{y_{+}}{2}\left(1-\sqrt{\frac{(a-1) y_{+}-3 A+1}{(a-1) y_{+}+A+1}}\right) \\
\phi_{-}=\frac{y_{-}}{2}\left(1+\sqrt{\frac{(a-1) y_{-}-3 A+1}{(a-1) y_{-}+A+1}}\right) \\
\psi_{-}=\frac{y_{-}}{2}\left(1-\sqrt{\frac{(a-1) y_{-}-3 A+1}{(a-1) y_{-}+A+1}}\right)\end{array}$ \\
\hline
\end{tabular}

Table 1: The conditions for existence of a minimal period-two solution(s)

where

$$
y_{ \pm}=\frac{-2+a+A \pm \sqrt{(2-a-A)^{2}+4(a-1)\left(1-A^{2}\right)}}{2(a-1)}, \quad \eta_{ \pm}(A)=\frac{2 A-1}{5 A-4} \pm 2 \frac{1-A}{5 A-4} \sqrt{1+\frac{1}{A}} .
$$




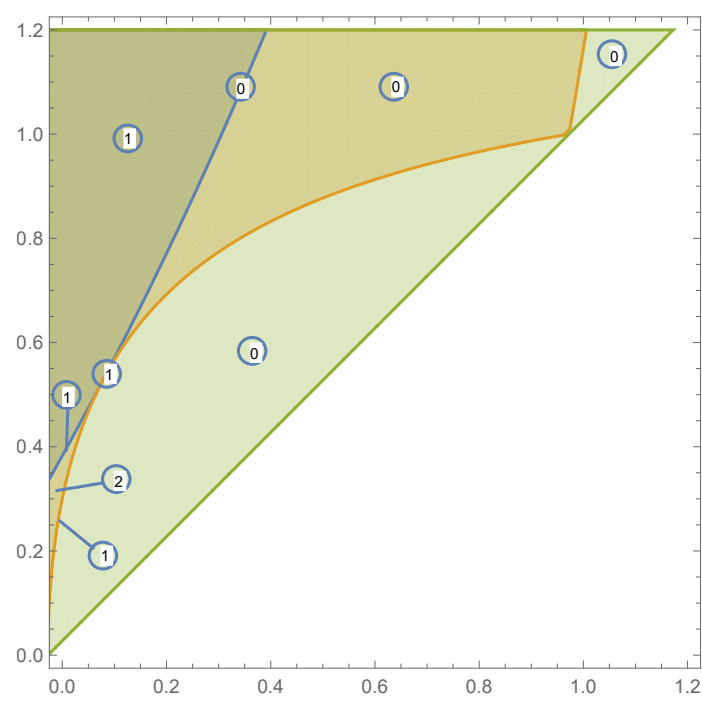

(a)

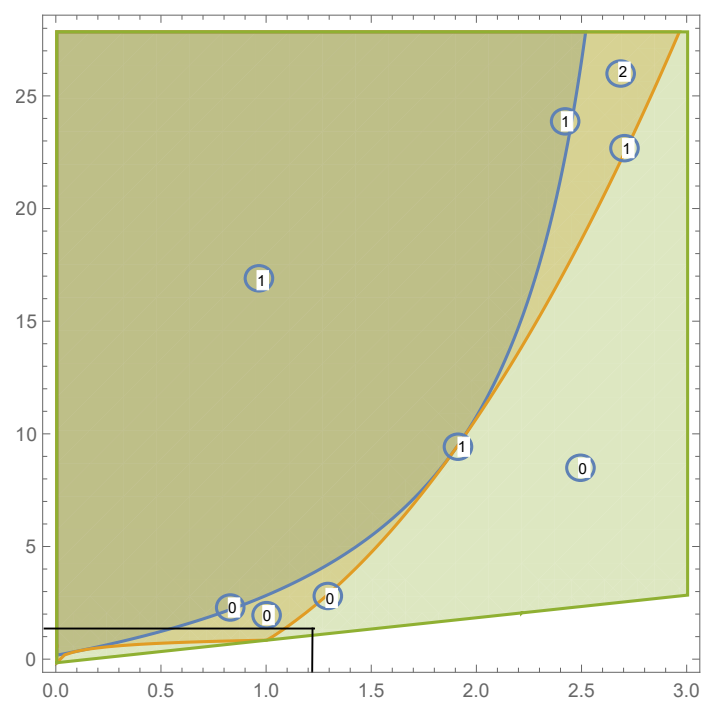

(b)

Figure 4.1: Visual representation of the areas that contain the minimal period-two solutions of Equation (2.1). The left image represents an enlarged rectangle region from the right image.

\subsection{Global Results}

In this section, we present the global dynamics of Equation (2.1) in some special cases.

Theorem 4.1. If $a=\frac{5 A+1}{3-A}$ and $A \in\left(0,1-\frac{2 \sqrt{5}}{5}\right] \cup\left[1+\frac{2 \sqrt{5}}{5}, 3\right)$, then Equation (2.1) has the unique equilibrium point $\bar{x}$ which is a nonhyperbolic point, and has the unique minimal period-two solution $\{\ldots \phi, \psi, \phi, \psi, \ldots\}$ of the form

$$
\phi=\frac{1}{2}\left(1+\sqrt{\frac{(3 A-1)(A-1)}{-A^{2}+8 A+1}}\right), \quad \psi=\frac{1}{2}\left(1-\sqrt{\frac{(3 A-1)(A-1)}{-A^{2}+8 A+1}}\right),
$$

which is locally asymptotically stable. There exists a set $\mathcal{C}=\mathcal{W}((\bar{x}, \bar{x}))$ which is the basin of attraction of $(\bar{x}, \bar{x})$. The set $\mathcal{C}$ is a graph of a strictly increasing continuous function of the first variable on an interval and separates $\mathcal{R}=([0, \infty) \times[0, \infty)) \backslash$ $\{(0,0)\}$ into two connected and invariant parts, $\mathcal{W}_{-}((\bar{x}, \bar{x}))$ and $\mathcal{W}_{+}((\bar{x}, \bar{x}))$, where

$$
\mathcal{W}_{-}((\bar{x}, \bar{x})):=\left\{(x, y) \in \mathcal{R} \backslash \mathcal{C}: \exists\left(x^{\prime}, y^{\prime}\right) \in \mathcal{C} \text { with }(x, y) \preceq_{\text {se }}\left(x^{\prime}, y^{\prime}\right)\right\}
$$

and

$$
\mathcal{W}_{+}((\bar{x}, \bar{x})):=\left\{(x, y) \in \mathcal{R} \backslash \mathcal{C}: \exists\left(x^{\prime}, y^{\prime}\right) \in \mathcal{C} \text { with }\left(x^{\prime}, y^{\prime}\right) \preceq_{s e}(x, y)\right\},
$$

such that

(i) if $\left(x_{-1}, x_{0}\right) \in \mathcal{W}_{-}((\bar{x}, \bar{x}))$, then $\lim _{n \rightarrow \infty} x_{2 n}=\phi$ and $\lim _{n \rightarrow \infty} x_{2 n+1}=\psi$;

(ii) if $\left(x_{-1}, x_{0}\right) \in \mathcal{W}_{+}((\bar{x}, \bar{x}))$, then $\lim _{n \rightarrow \infty} x_{2 n}=\psi$ and $\lim _{n \rightarrow \infty} x_{2 n+1}=\phi$. 
Proof. Since $y_{+}=1$ for $a=\frac{5 A+1}{3-A}$ and by using (4.4), we can see that Equation (2.1) has the unique minimal period-two solution $(\phi, \psi)$ of the form (4.12).

By substitution

$$
x_{n-1}=u_{n}, x_{n}=v_{n},
$$

Equation (2.1) becomes the system

$$
\begin{gathered}
u_{n+1}=v_{n}, \\
v_{n+1}=\frac{A v_{n}^{2}+u_{n}^{2}}{a v_{n}^{2}+u_{n}^{2}} .
\end{gathered}
$$

The map $T$ corresponding to (4.13) is of the form

$$
T\left(\begin{array}{l}
u \\
v
\end{array}\right)=\left(\begin{array}{c}
v \\
g(u, v)
\end{array}\right)
$$

where $g(u, v)=\frac{A v^{2}+u^{2}}{a v^{2}+u^{2}}$. The second iteration of the map $T$ is

$$
T^{2}\left(\begin{array}{l}
u \\
v
\end{array}\right)=T\left(\begin{array}{c}
v \\
g(u, v)
\end{array}\right)=\left(\begin{array}{c}
g(u, v) \\
g(v, g(u, v))
\end{array}\right)=\left(\begin{array}{c}
F(u, v) \\
G(u, v)
\end{array}\right)
$$

where

$$
F(u, v)=g(u, v) \text { and } G(u, v)=\frac{A F^{2}(u, v)+v^{2}}{a F^{2}(u, v)+v^{2}},
$$

and the map $T^{2}$ is strictly competitive, see $[4,5,9]$.

The Jacobian matrix of the map $T^{2}$ is

$$
J_{T^{2}}\left(\begin{array}{l}
u \\
v
\end{array}\right)=\left(\begin{array}{ll}
\frac{\partial F}{\partial u} & \frac{\partial F}{\partial v} \\
\frac{\partial G}{\partial u} & \frac{\partial G}{\partial v}
\end{array}\right),
$$

where

$$
\begin{aligned}
\frac{\partial F}{\partial u} & =\frac{2(a-A) u v^{2}}{\left(a v^{2}+u^{2}\right)^{2}}, \frac{\partial F}{\partial v}=-\frac{2(a-A) u^{2} v}{\left(a v^{2}+u^{2}\right)^{2}}, \frac{\partial G}{\partial u}=\frac{-2(a-A) v^{2} F(u, v) \frac{\partial F}{\partial u}}{\left(a F^{2}(u, v)+v^{2}\right)^{2}} \\
\frac{\partial G}{\partial v} & =\frac{2(a-A) v F(u, v)\left(F(u, v)-v \frac{\partial F}{\partial v}\right)}{\left(a F^{2}(u, v)+v^{2}\right)^{2}} .
\end{aligned}
$$

Notice that

$$
\phi=\frac{A \psi^{2}+\phi^{2}}{a \psi^{2}+\phi^{2}}, \quad \psi=\frac{A \phi^{2}+\psi^{2}}{a \phi^{2}+\psi^{2}}, \quad F(\phi, \psi)=\phi
$$

and

$$
\frac{\partial F}{\partial u}\left(\begin{array}{c}
\phi \\
\psi
\end{array}\right)=\frac{2(a-A) \phi \psi^{2}}{\left(a \psi^{2}+\phi^{2}\right)^{2}}, \frac{\partial F}{\partial v}\left(\begin{array}{c}
\phi \\
\psi
\end{array}\right)=-\frac{2(a-A) \phi^{2} \psi}{\left(a \psi^{2}+\phi^{2}\right)^{2}}
$$




$$
\begin{aligned}
& \frac{\partial G}{\partial u}\left(\begin{array}{c}
\phi \\
\psi
\end{array}\right)=-\frac{2(a-A) \psi^{2} F(\phi, \psi) \frac{\partial F}{\partial u}}{\left(a F^{2}(\phi, \psi)+\psi^{2}\right)^{2}}=\frac{-4(a-A)^{2} \phi^{2} \psi^{4}}{\left(a \phi^{2}+\psi^{2}\right)^{2}\left(a \psi^{2}+\phi^{2}\right)^{2}} \\
& \frac{\partial G}{\partial v}\left(\begin{array}{c}
\phi \\
\psi
\end{array}\right)=\frac{2(a-A) \phi \psi\left(\phi-\psi \frac{\partial F}{\partial v}\right)}{\left(a \phi^{2}+\psi^{2}\right)^{2}}=\frac{2(a-A) \phi \psi\left(\phi+\psi \frac{2(a-A) \phi^{2} \psi}{\left(a \psi^{2}+\phi^{2}\right)^{2}}\right)}{\left(a \phi^{2}+\psi^{2}\right)^{2}}
\end{aligned}
$$

Then the Jacobian matrix of the map $T^{2}$ at the point $(\phi, \psi)$ is of the form

$$
J_{T^{2}}\left(\begin{array}{c}
\phi \\
\psi
\end{array}\right)=\left(\begin{array}{cc}
\frac{2(a-A) \phi \psi^{2}}{\left(a \psi^{2}+\phi^{2}\right)^{2}} & -\frac{2(a-A) \phi^{2} \psi}{\left(a \psi^{2}+\phi^{2}\right)^{2}} \\
\frac{-4(a-A)^{2} \phi^{2} \psi^{4}}{\left(a \phi^{2}+\psi^{2}\right)^{2}\left(a \psi^{2}+\phi^{2}\right)^{2}} & \frac{2(a-A) \phi \psi\left(\phi+\psi \frac{2(a-A) \phi^{2} \psi}{\left(a \psi^{2}+\phi^{2}\right)^{2}}\right)}{\left(a \phi^{2}+\psi^{2}\right)^{2}}
\end{array}\right) .
$$

The corresponding characteristic equation is $\lambda^{2}-p \lambda+q=0$, where

$$
p=\frac{2(a-A) \phi \psi^{2}}{\left(a \psi^{2}+\phi^{2}\right)^{2}}+\frac{2(a-A) \phi \psi\left(\phi+\psi \frac{2(a-A) \phi^{2} \psi}{\left(a \psi^{2}+\phi^{2}\right)^{2}}\right)}{\left(a \phi^{2}+\psi^{2}\right)^{2}}>0
$$

and

$$
q=\frac{4(a-A)^{2} \phi^{3} \psi^{3}}{\left(a \psi^{2}+\phi^{2}\right)^{2}\left(a \phi^{2}+\psi^{2}\right)^{2}} .
$$

We need to show that $|p|<1+q$ and $q<1$. Namely,

$$
\begin{gathered}
|p|<1+q \Longleftrightarrow p<1+q \\
\Longleftrightarrow \frac{2(a-A) \phi \psi^{2}}{\left(a \psi^{2}+\phi^{2}\right)^{2}}+\frac{2(a-A) \phi \psi\left(\phi+\frac{2(a-A) \phi^{2} \psi^{2}}{\left(a \psi^{2}+\phi^{2}\right)^{2}}\right)}{\left(a \phi^{2}+\psi^{2}\right)^{2}}<1+\frac{4(a-A)^{2} \phi^{3} \psi^{3}}{\left(a \psi^{2}+\phi^{2}\right)^{2}\left(a \phi^{2}+\psi^{2}\right)^{2}} \\
\Longleftrightarrow \frac{2(a-A) \phi \psi^{2}}{\left(a \psi^{2}+\phi^{2}\right)^{2}}+\frac{2(a-A) \phi^{2} \psi}{\left(a \phi^{2}+\psi^{2}\right)^{2}}<1 \\
\Longleftrightarrow 2(-3+A) A(1+A)^{2}\left(-1-8 A+A^{2}\right) \frac{\left(3+3 A+2 A^{2}+130 A^{3}-69 A^{4}+59 A^{5}\right)}{\left(1+9 A+38 A^{2}-42 A^{3}+153 A^{4}-31 A^{5}\right)^{2}}<1 \\
\Longleftrightarrow\left\{\begin{array}{c}
(1-A)\left(1+A-34 A^{2}+152 A^{3}+10 A^{4}-7726 A^{5}-1084 A^{6}\right. \\
\left.-14960 A^{7}+9181 A^{8}-2043 A^{9}+118 A^{10}\right)>0
\end{array}\right\},
\end{gathered}
$$

which is satisfied for $A \in\left(0,1-\frac{2 \sqrt{5}}{5}\right] \cup\left[1+\frac{2 \sqrt{5}}{5}, 3\right)$.

On the other hand,

$$
\begin{aligned}
q<1 & \Longleftrightarrow \frac{4(a-A)^{2} \phi^{3} \psi^{3}}{\left(a \psi^{2}+\phi^{2}\right)^{2}\left(a \phi^{2}+\psi^{2}\right)^{2}}<1 \\
& \Longleftrightarrow\left(A \psi^{2}+\phi^{2}\right)^{2}\left(A \phi^{2}+\psi^{2}\right)^{2}>4(a-A)^{2} \phi^{5} \psi^{5}
\end{aligned}
$$




$$
\begin{aligned}
& \Longleftrightarrow \frac{4(-3+A)^{3} A^{3}(1+A)^{4}\left(-1-8 A+A^{2}\right)}{\left(1+9 A+38 A^{2}-42 A^{3}+153 A^{4}-31 A^{5}\right)^{2}}<1 \\
& \Longleftrightarrow\left\{\begin{array}{c}
4(3-A)^{3} A^{3}\left(1+A^{4}\right)\left(-1-8 A+A^{2}\right) \\
+\left(1+9 A+38 A^{2}-42 A^{3}+153 A^{4}-31 A^{5}\right)>0
\end{array}\right\},
\end{aligned}
$$

which is satisfied for $A \in\left(0,1-\frac{2 \sqrt{5}}{5}\right] \cup\left[1+\frac{2 \sqrt{5}}{5}, 3\right)$.

The Jacobian matrix of $T^{2}$ at the point $(\bar{x}, \bar{x})$ has the real eigenvalues $\lambda=\frac{1}{4}$ and $\mu=1$, and the eigenspace $E^{\lambda}=\left[\begin{array}{l}1 \\ 2\end{array}\right]$ associated with $\lambda$ is not a coordinate axis. Now, Theorem 2 from [4] completes the proof (for details see [4, Theorem 14]).

In the special case, when $1=A<a$, we have that $F=1-a<0$ implies $\operatorname{sgn} F=-\operatorname{sgn}(a-1)$ and inequality (4.7) is satisfied for $a>3$. Then we have similar results as in previous theorem.

Theorem 4.2. i) If $A=1<a \leq 3$, then Equation (2.1) has no minimal periodtwo solutions, and has the unique equilibrium point $\bar{x}$ which is globally asymptotically stable.

ii) If $A=1$ and $a>3$, then Equation (2.1) has the unique equilibrium point $\bar{x}$, which is a saddle point, and has the following unique minimal period-two solution $\{\ldots \phi, \psi, \phi, \psi, \ldots\}$, where

$$
\phi=\frac{1}{2}\left(1+\sqrt{\frac{a-3}{a+1}}\right), \quad \psi=\frac{1}{2}\left(1-\sqrt{\frac{a-3}{a+1}}\right),
$$

which is locally asymptotically stable. There exists a set $\mathcal{C}=\mathcal{W}((\bar{x}, \bar{x}))$ which is the basin of attraction of $(\bar{x}, \bar{x})$. The set $\mathcal{C}$ is a graph of a strictly increasing continuous function of the first variable on an interval and separates $([0, \infty) \times[0, \infty)) \backslash\{(0,0)\}$ into two connected and invariant parts, $\mathcal{W}_{-}((\bar{x}, \bar{x}))$ and $\mathcal{W}_{+}((\bar{x}, \bar{x}))$, such that

(i) if $\left(x_{-1}, x_{0}\right) \in \mathcal{W}_{-}((\bar{x}, \bar{x}))$, then $\lim _{n \rightarrow \infty} x_{2 n}=\phi$ and $\lim _{n \rightarrow \infty} x_{2 n+1}=\psi$;

(ii) if $\left(x_{-1}, x_{0}\right) \in \mathcal{W}_{+}((\bar{x}, \bar{x}))$, then $\lim _{n \rightarrow \infty} x_{2 n}=\psi$ and $\lim _{n \rightarrow \infty} x_{2 n+1}=\phi$.

Proof. i) It follows by using Theorem 2.2.

ii) By substitution

$$
x_{n-1}=u_{n}, x_{n}=v_{n},
$$

Equation (2.1) becomes the system

$$
\begin{gathered}
u_{n+1}=v_{n}, \\
v_{n+1}=\frac{v_{n}^{2}+u_{n}^{2}}{a v_{n}^{2}+u_{n}^{2}} .
\end{gathered}
$$


The second iteration of the map $T$ corresponding to (4.16) is of the form

$$
T^{2}\left(\begin{array}{l}
u \\
v
\end{array}\right)=T\left(\begin{array}{c}
v \\
g(u, v)
\end{array}\right)=\left(\begin{array}{c}
g(u, v) \\
g(v, g(u, v))
\end{array}\right)=\left(\begin{array}{c}
F(u, v) \\
G(u, v)
\end{array}\right)
$$

where

$$
F(u, v)=g(u, v)=\frac{v^{2}+u^{2}}{a v^{2}+u^{2}}, G(u, v)=\frac{F^{2}(u, v)+v^{2}}{a F^{2}(u, v)+v^{2}},
$$

and the map $T^{2}$ is competitive.

The Jacobian matrix of the map $T^{2}$ at the point $(\phi, \psi)$ is of the form

$$
J_{T^{2}}\left(\begin{array}{c}
\phi \\
\psi
\end{array}\right)=\left(\begin{array}{cc}
\frac{2(a-1) \phi^{3} \psi^{2}}{\left(\psi^{2}+\phi^{2}\right)^{2}} & \frac{-2 \phi^{4} \psi(a-1)}{\left(\psi^{2}+\phi^{2}\right)^{2}} \\
-\frac{4 \phi^{4} \psi^{6}(a-1)^{2}}{\left(\phi^{2}+\psi^{2}\right)^{4}} & \frac{2(a-1) \phi^{2} \psi^{3}}{\left(\psi^{2}+\phi^{2}\right)^{2}}+\frac{4(a-1)^{2} \phi^{5} \psi^{5}}{\left(\psi^{2}+\phi^{2}\right)^{4}}
\end{array}\right) .
$$

The corresponding characteristic equation is $\lambda^{2}-p \lambda+q=0$, where

$$
p=\operatorname{Tr} J_{T^{2}}\left(\begin{array}{c}
\phi \\
\psi
\end{array}\right)=\frac{2(a-1) \phi^{2} \psi^{2}(\phi+\psi)}{\left(\psi^{2}+\phi^{2}\right)^{2}}+\frac{4(a-1)^{2} \phi^{5} \psi^{5}}{\left(\psi^{2}+\phi^{2}\right)^{4}}>0
$$

and

$$
q=\operatorname{det} J_{T^{2}}\left(\begin{array}{c}
\phi \\
\psi
\end{array}\right)=\frac{4(a-1)^{2} \phi^{5} \psi^{5}}{\left(\psi^{2}+\phi^{2}\right)^{4}}>0
$$

Notice that

$$
\phi \psi=\frac{1}{a+1}, \psi^{2}+\phi^{2}=\frac{a-1}{a+1}, \phi+\psi=1 .
$$

We need to show that $|p|<1+q$ and $q<1$. Namely,

$$
\begin{aligned}
|p| & <1+q \Longleftrightarrow p<1+q \\
& \Longleftrightarrow \frac{2(a-1) \phi^{2} \psi^{2}(\phi+\psi)}{\left(\psi^{2}+\phi^{2}\right)^{2}}+\frac{4(a-1)^{2} \phi^{5} \psi^{5}}{\left(\psi^{2}+\phi^{2}\right)^{4}}<1+\frac{4(a-1)^{2} \phi^{5} \psi^{5}}{\left(\psi^{2}+\phi^{2}\right)^{4}} \\
& \Longleftrightarrow \frac{2(a-1) \phi^{2} \psi^{2}(\phi+\psi)}{\left(\psi^{2}+\phi^{2}\right)^{2}}<1 \stackrel{(4.17)}{\Longleftrightarrow} \frac{2(a-1) \frac{1}{(a+1)^{2}}}{\frac{(a-1)^{2}}{(a+1)^{2}}}<1 \\
& \Longleftrightarrow \frac{2}{a-1}<1 \Longleftrightarrow a>3,
\end{aligned}
$$

which is true.

Furthermore,

$$
q<1 \Longleftrightarrow \frac{4(a-1)^{2} \phi^{5} \psi^{5}}{\left(\psi^{2}+\phi^{2}\right)^{4}}<1 \Longleftrightarrow \frac{4(a-1)^{2}\left(\frac{1}{(a+1)}\right)^{5}}{\left(\frac{a-1}{a+1}\right)^{4}}<1 \Longleftrightarrow \frac{4 \frac{1}{a+1}}{(a-1)^{2}}<1
$$




$$
\Longleftrightarrow \frac{4}{(a+1)(a-1)^{2}}<1 \Longleftrightarrow a^{3}-a^{2}-a-3>0,
$$

which is true for $a>3$.

Now, Theorems 2.3 and 2.4 complete the proof (see the proof of Theorem 13 in [4]).

Visualization of Theorem $4.2 \mathrm{ii}$ ) is given in the Figure 4.2.

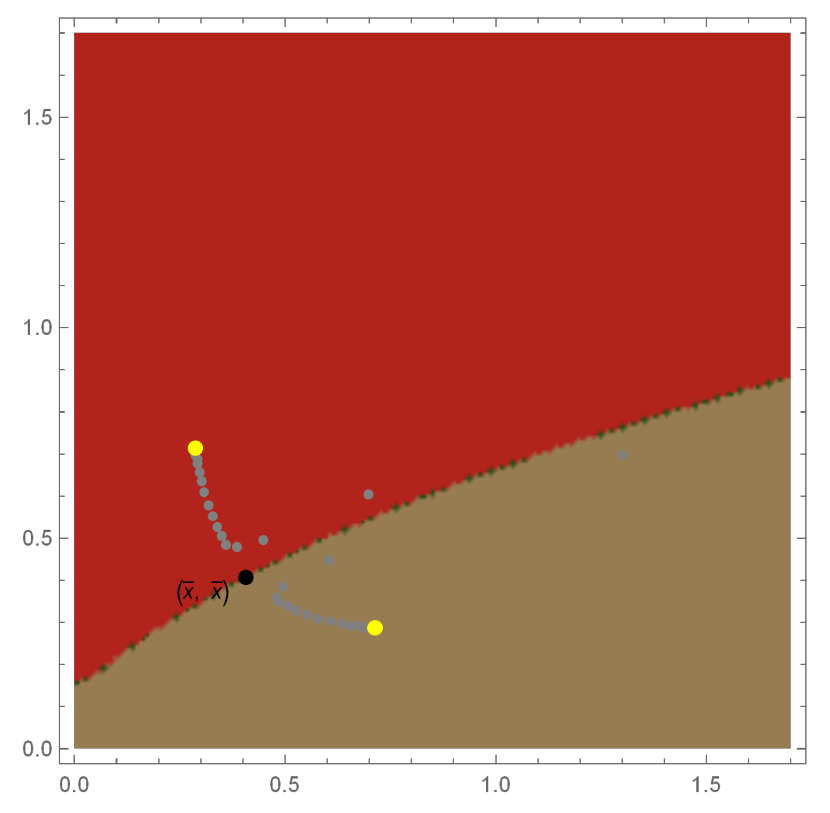

Figure 4.2: Basins of attraction for Equation (2.1) if $A=1$ and $a=3.9$ with initial conditions $\left(x_{-1}, x_{0}\right)=(1.3,0.7)-$ gray. Yellow points represent minimal period-two solutions $(\phi, \psi)$ and $(\psi, \phi)$.

Theorem 4.3. If $(A, a) \in\left(\Theta_{1} \cup \Theta_{2} \cup \Theta_{3}\right) \cap \Theta_{4}$, where

$$
\begin{aligned}
\Theta_{1} & =\left\{(A, a) \mid A \leq \frac{1}{3} \wedge 1<a\right\}, \\
\Theta_{2} & =\left\{(A, a) \mid A \in\left(\frac{1}{3}, 1\right) \cup(1,3) \wedge \frac{5 A+1}{3-A}<a\right\}, \\
\Theta_{3} & =\left\{(A, a) \mid A<\frac{1}{3} \wedge \frac{5 A+1}{3-A}<a<1\right\}, \\
\Theta_{4} & =\{(A, a) \mid A<a<9 A\}
\end{aligned}
$$

then Equation (2.1) has the unique equilibrium point $\bar{x}$, which is a saddle point, and has the unique minimal period-two solution of the form (4.4), which is locally asymptotically stable. There exists a set $\mathcal{C}=\mathcal{W}^{s}((\bar{x}, \bar{x}))$ which is the basin of attraction of $(\bar{x}, \bar{x})$. The 
set $\mathcal{C}$ is a graph of a strictly increasing continuous function of the first variable on an interval and separates $([0, \infty) \times[0, \infty)) \backslash\{(0,0)\}$ into two connected and invariant parts, $\mathcal{W}_{-}((\bar{x}, \bar{x}))$ and $\mathcal{W}_{+}((\bar{x}, \bar{x}))$, such that

(i) if $\left(x_{-1}, x_{0}\right) \in \mathcal{W}_{-}((\bar{x}, \bar{x}))$, then $\lim _{n \rightarrow \infty} x_{2 n}=\phi$ and $\lim _{n \rightarrow \infty} x_{2 n+1}=\psi$;

(ii) if $\left(x_{-1}, x_{0}\right) \in \mathcal{W}_{+}((\bar{x}, \bar{x}))$, then $\lim _{n \rightarrow \infty} x_{2 n}=\psi$ and $\lim _{n \rightarrow \infty} x_{2 n+1}=\phi$.

Proof. Notice that we can use Lemma 2.5 for the local stability analysis of the minimal period-two solution (4.15) (because we can not use the classical method as in proofs of two previous theorems). It is well known that $(\psi, \phi)$ and $(\phi, \psi)$ are the fixed points of the competitive map $T^{2}$ together with $(\bar{x}, \bar{x})$. The fixed points of the map $T^{2}$ are solutions of the following system

$$
T^{2}\left(\begin{array}{l}
x \\
y
\end{array}\right)=\left(\begin{array}{l}
x \\
y
\end{array}\right) \Longleftrightarrow\left(\begin{array}{l}
F(x, y) \\
G(x, y)
\end{array}\right)=\left(\begin{array}{l}
x \\
y
\end{array}\right) \Longleftrightarrow\left(\begin{array}{c}
F(x, y) \\
F(y, x)
\end{array}\right)=\left(\begin{array}{l}
x \\
y
\end{array}\right) .
$$

Let

$$
\begin{aligned}
& \mathcal{C}_{1}=\{(x, y): F(x, y)=x, x>0, y>0\}, \\
& \mathcal{C}_{2}=\{(x, y): F(y, x)=y, x>0, y>0\}
\end{aligned}
$$

from which implies that $\mathcal{C}_{1}$ is the graph of the function $y_{1}(x)=x \sqrt{\frac{1-x}{a x-A}}$ for $1 \geq x>\frac{A}{a}$ and $\mathcal{C}_{2}$ is the graph of the function $x(y)=y \sqrt{\frac{1-y}{a y-A}}$ for $1 \geq y>\frac{A}{a}$. If $y_{1}$ is injective, then $\mathcal{C}_{2}$ is the graph of the function $y_{2}(x), x \in[0, \infty)$, which is an inverse function of $y_{1}(x)$. It is easy to see that

$$
y_{1}^{\prime}(x)<0 \Longleftrightarrow-2 a x^{2}+(a+3 A) x-2 A<0 .
$$

If $A<a<9 A$ (because then is $\left.(a+3 A)^{2}-16 A=(a-A)(a-9 A)<0\right)$, the function $y_{1}(x)$ is a differentiable and strictly decreasing function on $\left(\frac{A}{a}, 1\right]$, which implies that $y_{2}(x)$ is differentiable and strictly decreasing function on $[0, \infty)$.

Let $m_{1}$ is the slope of the tangent to $\mathcal{C}_{1}$ at $(\psi, \phi)$ and $m_{2}$ is the slope of the tangent to $\mathcal{C}_{2}$ at $(\psi, \phi)$. It is clear from geometry that $m_{1}<m_{2}$ (see Figure 4.3 ), which implies that

$$
\left.\frac{d y}{d x}\right|_{\mathcal{C}_{1}}(\psi, \phi)<\left.\frac{d y}{d x}\right|_{\mathcal{C}_{2}}(\psi, \phi) .
$$

Since it is clear that the Jacobian matrix of $T^{2}$ at $(\psi, \phi)$ does not have a negative unstable eigenvalue, then Lemma 2.5 implies that $(\psi, \phi)$ is hyperbolic attractor. Also, by using the same conclusion we see that $(\phi, \psi)$ hyperbolic attractor.

Now, Theorems 2.3 and 2.4 complete the proof.

Based on our many computer simulations, we believe that the previous theorem is valid if $(A, a) \in \Theta_{1} \cup \Theta_{2} \cup \Theta_{3}$. Also, we believe that the following two conjectures are true. 


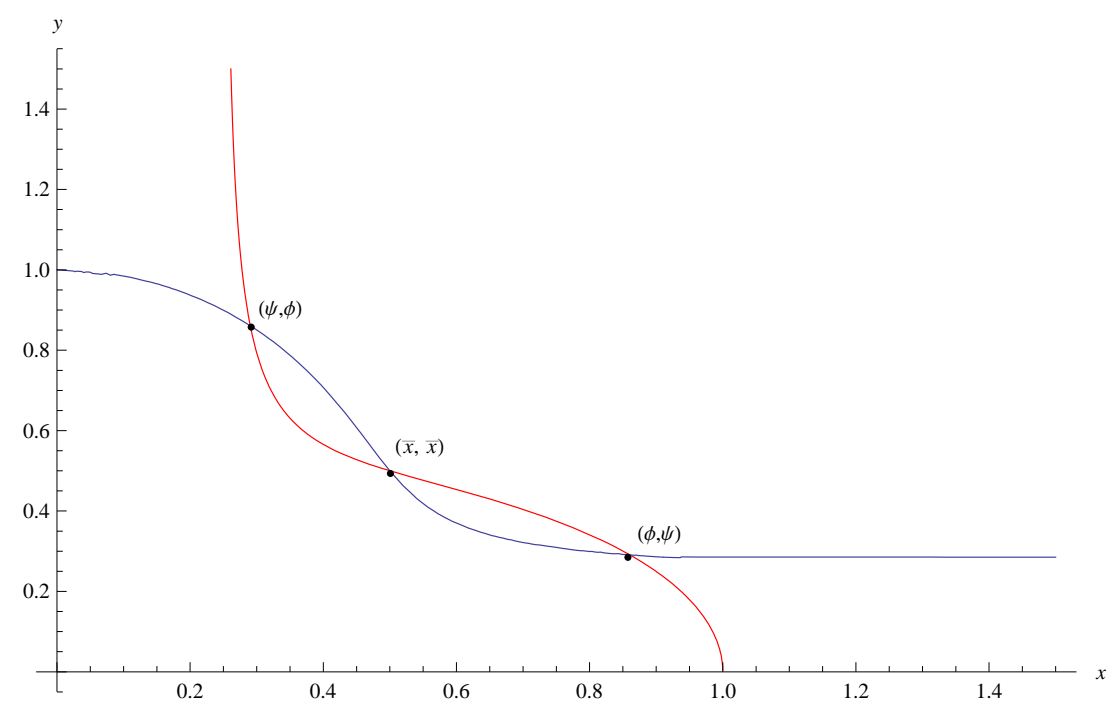

Figure 4.3: Graphs of the function $C_{1}$ (red) and its inverse function $C_{2}$ (blue).

Conjecture 4.4. Suppose that $a>A$. If any of the following conditions is satisfied

(i) $\left(A<1-\frac{2 \sqrt{5}}{5} \wedge \frac{3-A}{5 A+1}<\frac{1}{a}<\frac{2 A-1}{5 A-4}-2 \frac{1-A}{5 A-4} \sqrt{1+\frac{1}{A}}\right)$
(ii) $\left(A>1+\frac{2 \sqrt{5}}{5} \wedge \max \left\{0, \frac{3-A}{5 A+1}\right\}<\frac{1}{a}<\frac{2 A-1}{5 A-4}+2 \frac{1-A}{5 A-4} \sqrt{1+\frac{1}{A}}\right)$,

then Equation (2.1) has the unique equilibrium point $\bar{x}$, which is locally asymptotically stable, and has two minimal period-two solutions: (4.4) which is saddle point and (4.5) which is the locally asymptotically stable. The basin of attraction $\mathcal{B}((\bar{x}, \bar{x}))$ of $(\bar{x}, \bar{x})$ is the region between the global stable sets $\mathcal{W}^{s}\left(\left(\phi_{+}, \psi_{+}\right)\right)$and $\mathcal{W}^{s}\left(\left(\psi_{+}, \phi_{+}\right)\right)$. The basins of attraction $\mathcal{B}\left(\left(\phi_{+}, \psi_{+}\right)\right)=\mathcal{W}^{s}\left(\left(\phi_{+}, \psi_{+}\right)\right)$and $\mathcal{B}\left(\left(\psi_{+}, \phi_{+}\right)\right)=\mathcal{W}^{s}\left(\left(\psi_{+}, \phi_{+}\right)\right)$are exactly the global stable sets of $(\phi, \psi)$ and $(\psi, \phi)$. Furthermore, the basin of attraction of the minimal period-two solution $\left(\phi_{-}, \psi_{-}\right)$( or $\left.\left(\psi_{-}, \phi_{-}\right)\right)$is the region between the global stable sets $\mathcal{W}^{s}\left(\left(\phi_{-}, \psi_{-}\right)\right)\left(\mathcal{W}^{s}\left(\left(\psi_{-}, \phi_{-}\right)\right)\right)$and the coordinate axis.

Conjecture 4.4 has its visual confirmation if, for example, $A=0.01$ and $a=0.25$. See Figure 4.4.

Conjecture 4.5. Suppose that $a>A$. If any of the following conditions is satisfied

$$
\begin{aligned}
& \text { (i) }\left(A<1-\frac{2 \sqrt{5}}{5} \wedge \frac{1}{a}=\frac{2 A-1}{5 A-4}-2 \frac{1-A}{5 A-4} \sqrt{1+\frac{1}{A}}\right), \\
& \text { (ii) }\left(A>1+\frac{2 \sqrt{5}}{5} \wedge \frac{1}{a}=\frac{2 A-1}{5 A-4}+2 \frac{1-A}{5 A-4} \sqrt{1+\frac{1}{A}}\right)
\end{aligned}
$$




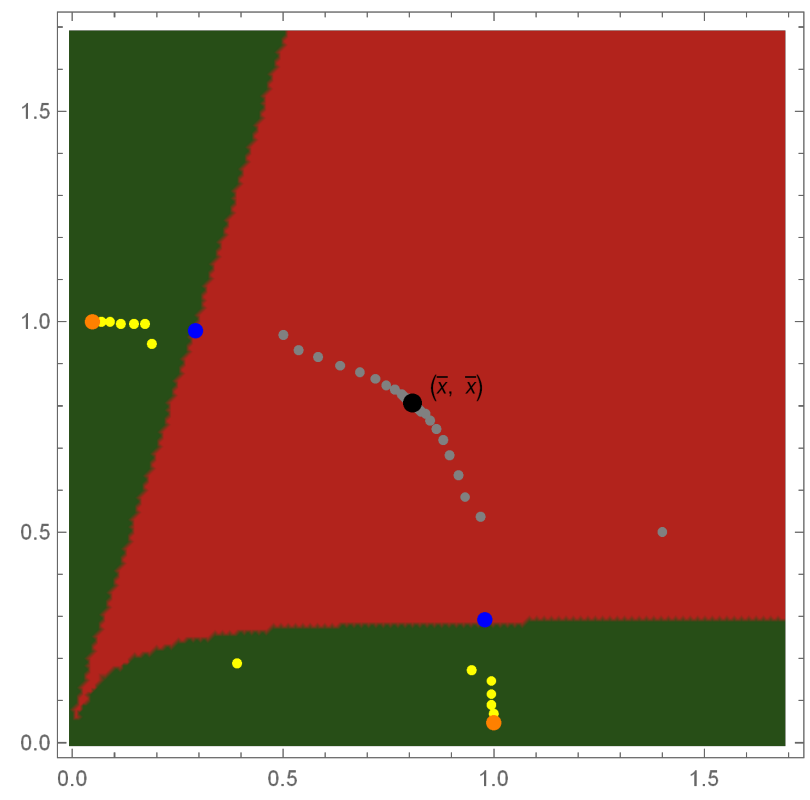

Figure 4.4: Basins of attraction for (2.1) if $A=0.01$ and $a=0.25$ with initial conditions $\left(x_{-1}, x_{0}\right)=(1.4,0.5)-$ gray, $\left(x_{-1}, x_{0}\right)=(0.39,0.19)-$ yellow. Blue points represent the minimal period-two solutions $\left(\phi_{-}, \psi_{-}\right)$and $\left(\psi_{-}, \phi_{-}\right)$and orange points represent the minimal period-two solutions $\left(\phi_{+}, \psi_{+}\right)$and $\left(\psi_{+}, \phi_{+}\right)$.

then Equation (2.1) has the unique equilibrium point $\bar{x}$, which is locally asymptotically stable, and has the unique minimal period-two solution $(\phi, \psi)$, which is nonhyperbolic. The Jacobian matrix of $T^{2}$ at the $(\phi, \psi)$ has real eigenvalues $\lambda, \mu$ such that $0<|\lambda|<\mu$, where $|\lambda|<1$, and the eigenspace $E^{\lambda}$ associated with $\lambda$ is not a coordinate axis. Then there exists a curve $\mathcal{C}_{1}\left(\mathcal{C}_{2}\right) \subset([0, \infty) \times[0, \infty)) \backslash\{(0,0)\}$ through $(\phi, \psi)((\psi, \phi))$ that is invariant and a subset of the basin of attraction of $(\phi, \psi)((\psi, \phi))$, such that $\mathcal{C}_{1}\left(\mathcal{C}_{2}\right)$ is tangential to the eigenspace $E^{\lambda}$ at $(\phi, \psi)((\psi, \phi))$, and $\mathcal{C}_{1}\left(\mathcal{C}_{2}\right)$ is the graph of a strictly increasing continuous function of the first coordinate on an interval where one endpoint is origin and another is infinity.

Furthermore, the basin of attraction $\mathcal{B}((\bar{x}, \bar{x}))$ of $(\bar{x}, \bar{x})$ is the region between the global stable sets $\mathcal{C}_{1}$ and $\mathcal{C}_{2}$. The basins of attraction of the minimal period-two solutions $(\phi, \psi)((\psi, \phi))$ is the region between the sets $\mathcal{C}_{1}\left(\mathcal{C}_{2}\right)$ and the coordinate axis.

For example, the behavior described in the Conjecture 4.5 we have in the Equation (2.1) with parameters $A=2$ and $a=6+2 \sqrt{6}$. See Figure 4.5.

Next result follows from Theorem 2.2.

Theorem 4.6. Suppose that a $>$ A. In all cases where Equation (2.1) has no minimal period-two solutions, the unique equilibrium point $\bar{x}$ is globally asymptotically stable. 


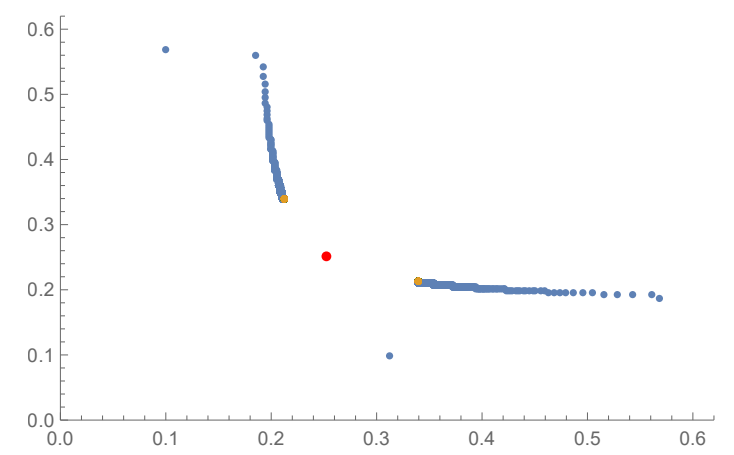

(a)

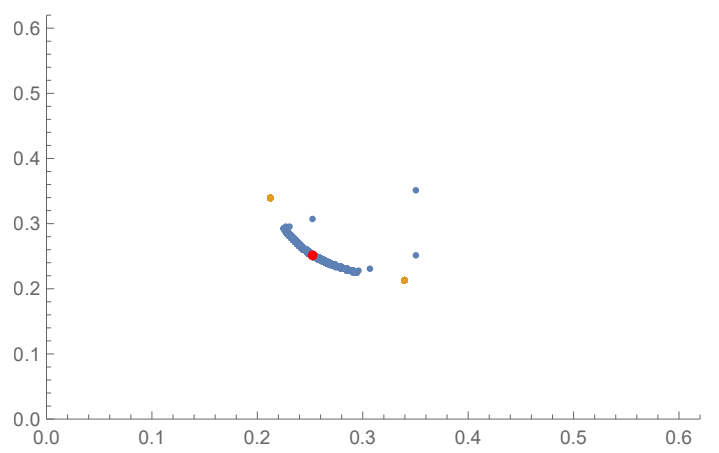

(b)

Figure 4.5: The phase portrait for values of parameters $A=2, a=6+2 \sqrt{6}$ and initial conditions (a) $\left(x_{-1}, x_{0}\right)=(0.312,0.1)$, (b) $\left(x_{-1}, x_{0}\right)=(0.35,0.35)$.

\section{Acknowledgements}

This paper was supported in part by FMON of Bosnia and Herzegovina number 01/25240-1/18.

\section{References}

[1] A. M. Amleh, E. Camouzis, and G. Ladas, On the Dynamics of a Rational Difference Equation, Part I, Int. J. Difference Equ., 3 (2008), 1-35.

[2] A. M. Amleh, E. Camouzis, and G. Ladas, On the Dynamics of a Rational Difference Equation, Part II, Int. J. Difference Equ., 3 (2008), 195-225.

[3] E. Camouzis and G. Ladas, Dynamics of third-order rational difference equations with open problems and conjectures. Advances in Discrete Mathematics and Applications, vol. 5. Chapman \& Hall/CRC, Boca Raton, FL, 2008.

[4] M. Garić-Demirović, M. R. S. Kulenović and M. Nurkanović, Global Dynamics of Certain Homogeneous Second-Order Quadratic Fractional Difference Equations, The Scientific World Journal, Volume 2013 (2013), Article ID 210846, 10 pages.

[5] M. Garić-Demirović, M. R. S. Kulenović and M. Nurkanović, Basins of Attraction of Certain Homogeneous Second Order Quadratic Fractional Difference Equation, Journal of Concrete and Applicable Mathematics, Vol. 13 (2015), 35-50.

[6] M. Garić-Demirović, M. R. S. Kulenović and M. Nurkanović, Global behavior of four competitive rational systems of difference equations in the plane, Discrete Dynamics in Nature and Society, Vol. 2009 (2009), Article ID 153058, 34 pages. 
[7] M. Garić-Demirović, M. R. S. Kulenović and M. Nurkanović, Global Behavior of Two Competitive Rational Systems of difference Equations in the Plane, Communications on Applied Nonlinear Analysis, Vol. 16 (3) (2009),1-18.

[8] M. Garić-Demirović, M. Nurkanović and Z. Nurkanović, Stability, Periodicity and Neimark-Sacker bifurcation of Certain Homogeneous Fractional Difference Equation, International Journal of Difference Equations ISSN 0973-6069, Vol. 12(1) (2017), 27-53.

[9] S. Jašarević Hrustić, M.R.S. Kulenović and M. Nurkanović, Global Dynamics and Bifurcations of Certain Second Order Rational Difference Equation with Quadratic Terms, Qualitative Theory of Dynamical Systems, Vol. 15 (2016), 283307.

[10] T. Khyat, M.R.S. Kulenović and E. Pilav, The Naimark-Sacker bifurcation and asymptotic approximation of the invariant curve of a certain difference equation, J. Comp. Anal. Appl., Vol. 23, No.8 (2017), 1335-1346.

[11] M. R. S. Kulenović and G. Ladas, Dynamics of Second Order Rational Difference Equations with Open Problems and Conjectures, Chapman and Hall/CRC, Boca Raton, London, 2001.

[12] M. R. S. Kulenović, G. Ladas and W. Sizer, On the recursive sequence $x_{n+1}=$ $\left(\alpha x_{n}+\beta x_{n-1}\right) /\left(\gamma x_{n}+\delta x_{n-1}\right)$, Math. Sci. Res. Hot/line 2 (1998), 1-16.

[13] M. R. S. Kulenović and O. Merino, Discrete Dynamical Systems and Difference Equations with Mathematica, Chapman and Hall/CRC, Boca Raton, London, 2002.

[14] M. R. S. Kulenović and O. Merino, Invariant manifolds for planar competitive and cooperative maps, Journal of Difference Equations and Applications, Vol. 24 (6) (2018), 898-915.

[15] M.R.S. Kulenović, O. Merino and M. Nurkanović, Global dynamics of certain competitive system in the plane, Journal of Difference Equations and Applications, Vol. 18 (12) (2012), 1951-1966.

[16] M.R.S. Kulenović, S. Moranjkić and Z. Nurkanović, Global dynamics and bifurcation of perturbed Sigmoid Beverton-Holt difference equation, Math. Meth. Appl. Sci., 39 (2016), 2696-2715.

[17] M. R. S. Kulenović and M. Nurkanović, Asymptotic Behavior of a Competitive System of Linear Fractional Difference Equations, Advances in Difference Equations, Vol.2006 (2006), Article ID 19756, 1-13. 
[18] M. R. S. Kulenović and M. Nurkanović, Global behavior of a two-dimensional competitive system of difference equations with stocking, Mathematical and Computer Modelling, 55 (2012), 1998-2011, doi:10.1016/j.mcm.2011.11.059.

[19] M. R. S. Kulenović and Z. Nurkanović, Global behavior of a three-dimensional linear fractional system of difference equations, Journal of Mathematical Analysis and Applications, Vol. 310 (2) (2005):673-689, DOI: 10.1016/j.jmaa.2005.02.042.

[20] S. Kalabušić, M. Nurkanović and Z. Nurkanovi ć, Global Dynamics of Certain Mix Monotone Difference Equations, Mathematics, 6 (1) (2018), 10.

[21] C. M. Kent and H. Sedaghat, Global attractivity in a quadratic-linear rational difference equation with delay, J. Difference Equ. Appl., 15 (2009), 913-925.

[22] C. M. Kent and H. Sedaghat, Global attractivity in a rational delay difference equation with quadratic terms, J. Difference Equ. Appl., 17 (2011), 457-466.

[23] S. Moranjkić and Z. Nurkanović, Local and Global Dynamics of Certain SecondOrder Rational Difference Equations Containing Quadratic Terms, Advances in Dynamical Systems and Applications, Vol. 12 (2) (2017), 123-157.

[24] S. Moranjkić and Z. Nurkanović, Basins of attraction of certain rational anticompetitive system of difference equations in the plane, Advances in Difference Equations, Vol. 2012 (2012), article 153.

[25] H. Sedaghat, Global behaviours of rational difference equations of orders two and three with quadratic terms, J. Difference Equ. Appl., 15 (2009), 215-224. 